\title{
Review Article \\ Fiber Optic Chemical Nanosensors Based on Engineered Single-Walled Carbon Nanotubes
}

\author{
M. Consales, ${ }^{1}$ A. Cutolo, ${ }^{1}$ M. Penza, ${ }^{2}$ P. Aversa, ${ }^{2}$ M. Giordano, ${ }^{3}$ and A. Cusano ${ }^{1}$ \\ ${ }^{1}$ Optoelectronic Division, Engineering Department, University of Sannio, C.so Garibaldi 107, 82100 Benevento, Italy \\ ${ }^{2}$ Dipartimento Tecnologie Fisiche e Nuovi Materiali, Ente per le Nuove Tecnologie, l'Energia e l'Ambiente (ENEA), \\ SS.7, Appia, $\mathrm{km}$ 714, 72100 Brindisi, Italy \\ ${ }^{3}$ Institute for Composite and Biomedical Materials, National Research Council, P.le Enrico Fermi 1, 80055 Portici, Italy \\ Correspondence should be addressed to M. Consales, consales@unisannio.it
}

Received 16 April 2008; Accepted 21 April 2008

Recommended by Ignacio Matias

In this contribution, a review of the development of high-performance optochemical nanosensors based on the integration of carbon nanotubes with the optical fiber technology is presented. The paper first provide an overview of the amazing features of carbon nanotubes and their exploitation as highly adsorbent nanoscale materials for gas sensing applications. Successively, the attention is focused on the operating principle, fabrication, and characterization of fiber optic chemosensors in the Fabry-Perot type reflectometric configuration, realized by means of the deposition of a thin layer of single-walled carbon nanotubes (SWCNTs) upon the distal end of standard silica optical fibers. This is followed by an extensive review of the excellent sensing capabilities of the realized SWCNTs-based chemical nanosensors against volatile organic compounds and other pollutants in different environments (air and water) and operating conditions (room temperature and cryogenic temperatures). The experimental results reported here reveal that ppm and sub-ppm chemical detection limits, low response times, as well as fast and complete recovery of the sensor responses have been obtained in most of the investigated cases. This evidences the great potentialities of the proposed photonic nanosensors based on SWCNTs to be successfully employed for practical environmental monitoring applications both in liquid and vapor phase as well as for space. Furthermore, the use of novel SWCNTs-based composites as sensitive fiber coatings is proposed to enhance the sensing performance and to improve the adhesion of carbon nanotubes to the fiber surface. Finally, new advanced sensing configurations based on the use of hollow-core optical fibers coated and partially filled by carbon nanotubes are also presented.

Copyright (c) 2008 M. Consales et al. This is an open access article distributed under the Creative Commons Attribution License, which permits unrestricted use, distribution, and reproduction in any medium, provided the original work is properly cited.

\section{INTRODUCTION}

Environmental monitoring is required to protect the public and the environment from toxic contaminants and pathogens that can be released into a variety of media including air, soil, and water. Air pollutants include sulfur dioxide, carbon monoxide, nitrogen dioxide, and volatile organic compounds (VOCs), which originate from sources such as vehicle emissions, power plants, refineries, and industrial and laboratory processes. Soil and water contaminants can be classified as microbiological, radioactive, inorganic, synthetic organic, and VOCs. Pesticides and herbicides are applied directly to plants and soils, and incidental releases of other contaminants can originate from spills, leaking pipes, underground storage tanks, waste dumps, and waste repositories. Some of these contaminants can persist for many years and migrate through large regions of soil until they reach water resources, where they may present an ecological or human-health threat. The current monitoring methods are mainly based on offsite laboratory analyses and are costly and time-consuming. In addition, limitations in sampling and analytical techniques occur $[1,2]$. For this reason, a need exists for accurate, inexpensive, continuous, and long-term monitoring of environmental contaminants using sensors that can be operated on site.

Chemical sensors can be in most cases schematically described as composed of a sensitive part which, interacting with the surrounding environment, collects and concentrates molecules at or within the surface undergoing physical changes, and of an opportune transducer which converts these changes of the sensing part into interpretable and quantifiable terms. The heart of the chemical sensor is the 
sensitive element which is the interface between transducer and external environment so that the nature, the selectivity, and sensitivity of the sensor depend upon these interactive materials. Good materials to use as sensing part should optimize specific interactions with a target analyte or narrow class of analytes that should provide a fast and reversible diffusion of the penetrants and small recovery times, and also they should maintain the physical state as well as their geometry over several cycles of use, in order to avoid hysteretic effects, and thus to ensure the reproducibility [3]. Candidate materials for chemical sensors include polymers, organic monolayers, ceramics, metals semiconductors, nanostructured and porous materials (nanomaterials, molecular sieves, sol-gels, aerogels), biomolecules, and combination thereof. Of course, adequate and detailed physical, chemical, and structural characterizations of the exploited sensing materials are essential for a deep understanding of their properties and for a rational design of the whole chemical sensor and of its performances.

The natural step following the selective recognition of an analyte from the sensitive layer is the signal transduction, and thus the choice of an opportune technique to read the physical or chemical changes occurring at the sensing part. Transducing approaches can include mechanical (acoustic wave, micromechanical), electrochemical, optical, thermal, and electronic types. Each has strengths and weaknesses relative to the particular application. Each transduction principle can be implemented in a variety of configurations, and fabricated by multiple approaches, resulting in many different sensing platforms.

A number of chemical sensors have been developed for environmental monitoring applications [4], a classification of which can be carried out upon their principal physics and operating mechanisms. The most exploited transduction principles in chemical sensing are the mass change and the resistivity/conductivity change of the sensitive element occurring on exposure to and consequent sorption of the molecules of target environmental analytes. The first physical parameter is in many cases measured by the shift in the resonant frequency of an oscillating piezoelectric crystal. Depending on the kind of vibrational wave propagated in the crystal, a mass sensor can be classified as quartz crystal microbalance (QCM) or surface acoustic wave (SAW) sensors [5-8]. They typically use a thin polymeric film as sensitive layer [9], however SAW and QCM-based chemical sensors using other sensitive coatings have been proposed $[10,11]$. Instead, resistivity/conductivity changes are typically detected by conductometric measurement carried out on sensitive materials (mainly semiconducting metal oxides and conjugated polymers) deposited between two electrodes. These sensor technologies carry the name of metal oxide semiconductors (MOS) and conductive organic polymer (COP) sensors [12]. During the last two decades, however, a remarkable interest has been also focused on optical transduction principles for the measurement of chemical and biological quantities [13]. Since the development of the first optical sensors for the measurement of $\mathrm{CO}_{2}$ and $\mathrm{O}_{2}$ concentrations [14], a large variety of devices based upon optical methods have been used in chemical sensors and biosensors including ellipsometry, spectroscopy (luminescence, phosphorescence, fluorescence, Raman), interferometry (white light interferometry, modal interferometry in optical waveguide structures), spectroscopy of guided modes in optical waveguide structures (grating coupler, resonant mirror), and surface plasmon resonance [15-25]. In these sensors, a desired quantity is determined by measuring the refractive index, absorbance, and fluorescence properties of analyte molecules or of a chemooptical transducing medium.

Optical fiber sensors are also very attractive in chemical sensing applications due to some unique characteristics derived by the use of optical fibers, one of the most outstanding characteristics of which is their ability to transmit light over large distances with low losses allowing a sensor head to be remotely located from the instrumentation. This feature is particularly useful for sensing in harsh environments where hazardous chemicals may be present or extremes in temperature occur. In addition to the small size, light weight and high flexibility of fibers allow access to areas that would be otherwise difficult to reach.

Provided the optical power density is within certain limits, fiber optic chemical sensors are much safer in explosive environments compared with sensors involving electrical signals, where a spark may trigger a gas explosion. Optical signals are immune to electrical or magnetic interference from, for example, power lines and electrical machinery. Furthermore, optical fibers have the capability of carrying a huge amount of information, much greater than that carried by electrical wires. Fiber optic sensing is very versatile, since the intensity, wavelength, phase, and polarization of light can all be exploited as measurement parameters, and several wavelengths launched in the same fiber in either direction form independent signals. This gives the possibility of monitoring several chemicals with the same fiber sensor or even simultaneously monitoring unwanted environment parameters variations which could drastically affect the chemical concentration measurements, such as the temperature or disturbance of the fiber. Multiplexing of fiber optic systems is also relatively easy, allowing expensive source or analysis instrumentation to be shared among a number of sites.

In this contribution, a review on the integration of carbon nanotubes as advanced nanostructured sensitive coatings with the optical fiber technology for the development of high-performance optochemical nanosensors to exploit for a wide range of environmental monitoring applications is carried out. In particular, the excellent sensing capabilities of the realized photonic chemosensors demonstrated against VOCs and other pollutants in different environments (air and water) and operating conditions (room temperature and cryogenic temperatures) will be reviewed. As it will be seen in the following sections, ppm and sub-ppm chemical detection limits have been obtained in most of the investigated cases.

\section{CARBON NANOTUBES AS ADVANCED MATERIALS FOR CHEMICAL SENSING}

The search for new advanced materials is an important area of contemporary research in numerous disciplines of science. 
Great attention has been paid in recent years to nanostructured materials of different chemical composition, produced as nanoparticles, nanowires, or nanotubes. Similarly, there has already been great interest in their preparation, properties, and applications in the literature. As a matter of fact, with the development of nanoscience and nanotechnology, a large number of literatures on one-dimensional nanostructured materials, including tubes, rods, belts, and wires in this area, have been published every year [26]. These materials have their unique structures which are dominated by a wire-like structure whose diameter varies over a broad range from several tens of nanometers to a micrometer. In particular, carbon-based nanostructures exhibit unique properties and morphological flexibility, which renders them inherently multifunctional and compatible with organic and inorganic systems.

Carbon nanotubes (CNTs), discovered by Iijima in 1991 [27], are at the forefront of the novel nanoscale investigations and nanostructure effects due to their unique electronic, chemical, structural, optical, mechanical, and thermal properties depending on their specific hollow nanostructure with surface-arranged carbon atoms organized in rolled onedimensional seamless tubes $[28,29]$. They are considered as a new form of pure carbon, and can essentially be thought of as layers of graphite rolled-up into a tube to form a cylinder with diameter of few nanometers and length ranging from 1 to 100 microns. To date, CNTs are building blocks considered as the most promising functional nanomaterial for future miniaturized gas nanosensors due to their hollow nanostructure and high specific surface area which provide attractive characteristics for gas sensing applications. In fact, due to their unique morphology, CNTs possess the excellent capability to reversibly adsorb molecules of environmental pollutants undergoing a modulation of their electrical, geometrical, and optical properties, such as conductivity, refractive index, and thickness. CNTs can be distinguished in SWCNTs or multiwalled carbon nanotubes (MWCNTs) depending on whether only one layer or many layers of graphite are concentrically rolled up together, and can behave either as metallic or semiconducting, depending upon their diameter and chirality (the way the hexagons are arranged along the tubule axis) [30]. SWCNTs are a very important variety of carbon nanotubes because they exhibit important electrical and sensing properties that are not shared by the MWCNTs variants. A further distinction is usually made between closed-end or opened-end CNTs depending upon whether the ends of the tubes are capped or uncapped [31].

\subsection{Chemical sensors based on carbon nanotubes: state of the art}

The special geometry of carbon nanotubes and their characteristic of being all surface reacting materials offer great potential applications as chemical sensor devices with excellent sensitivities and fast responses [32]. Most of the sensors based on CNTs are field effect transistors (FET), since much interest has been focused in the past on the study of the changes in their electrical properties as a consequence of the interaction with gaseous and VOC molecules: many studies have shown that although carbon nanotubes are robust and inert structures, their electrical properties are extremely sensitive to the effects of charge transfer and chemical doping by various molecules. The electronic structures of target molecules near the semiconducting nanotubes cause measurable changes to the nanotubes electrical conductivity. Nanosensors based on changes in electrical conductance are highly sensitive, but they are also limited by factors such as their inability to identify analytes with low adsorption energies, poor diffusion kinetics, and poor charge transfer with CNTs [33].

Kong et al. [34] were probably the first to show that CNTs can be used in chemical sensors since exposing SWCNTs to electron withdrawing (e.g., $\mathrm{NO}_{2}$ ) or donating (e.g., $\mathrm{NH}_{3}$ ) gaseous molecules dramatically increases or decreases the electrical resistance of SWCNTs in the transistor scheme. In addition, CNTs-based sensors demonstrated a fast response and a higher sensitivity than, for example, solid-state sensors at room temperature.

More or less in the same period, it was demonstrated that the electrical conductance of SWCNTs could be modified in presence of $\mathrm{O}_{2}$ [35]. The effect of the adsorption of several gas compounds in SWCNTs was also described [36], as well as those of water vapor on the electrical resistance of an SWCNT [37]. Shortly afterwards, Fujiwara et al. [38] studied the $\mathrm{N}_{2}$ and $\mathrm{O}_{2}$ adsorption properties of SWCNT bundles and their structures. All these studies opened the door to the development of chemical sensors based on CNTs.

Sensing devices based not only on the changes in the electrical properties of CNTs but also on other principles were proposed. For example, bundles of SWCNTs [39] have measured the thermoelectric qualitative response to a variety of gases $\left(\mathrm{He}, \mathrm{N}_{2}, \mathrm{H}_{2}, \mathrm{O}_{2}\right.$, and $\left.\mathrm{NH}_{3}\right)$. Sumanasekera et al. [40] created a thermoelectric chemical sensor to measure the easily detectable and reversible thermoelectric power changes of SWCNTs when they are in contact with $\mathrm{He}, \mathrm{N}_{2}$, and $\mathrm{H}_{2}$. Chopra et al. [41] developed a circular disk resonator coated with SWCNTs using a conductive epoxy, which selectively detects the qualitative presence of several gases $\left(\mathrm{NH}_{3}, \mathrm{CO}\right.$, Ar, $\mathrm{N}_{2}$, and $\mathrm{O}_{2}$ ) due to changes in the dielectric constant and shifts in the resonant frequency.

Wei et al. [42] demonstrated a gas sensor depositing CNT bundles onto a piezoelectric quartz crystal. This sensor detected $\mathrm{CO}, \mathrm{NO}_{2}, \mathrm{H}_{2}$, and $\mathrm{N}_{2}$ by detecting changes in oscillation frequency and was more effective at higher temperatures $\left(200^{\circ} \mathrm{C}\right)$. Penza et al. [43] developed SAW and QCM sensors coated with SWCNTs and MWCNTs and used them to detect VOCs such as ethanol, ethylacetate, and toluene by measuring the downshift in the resonance frequency of the acoustic transducers.

Carbon nanotubes can be easily functionalized with molecules enabling the specific interaction with target chemicals, thus improving the typically low selectivity of CNTsbased devices. In this way, different types of sensors based on molecular recognition interactions can be developed, allowing the development of nanosensors that are highly selective and sensitive. Chen et al. [44] used a noncovalent functionalized FET based on SWCNTs for selectively recognizing target proteins in solution. Azamian et al. [45] 
immobilized glucose oxidase on SWCNTs and enhanced the catalytic signal by more than one order of magnitude compared to that of an activated macrocarbon electrode. Zhao et al. [46] worked with horseradish peroxidase and Sotiropoulou et al. [47] worked with enzymes. Barone et al. [48] developed a device for $\beta$-D-glucose sensing in solution phase. They also showed two distinct mechanisms of signal transduction: fluorescence and charge transfer.

Recently, further interest has also been devoted to the possibility of changing the optical and/or geometrical properties of SWCNTs upon adsorption of target analyte molecules, enabling to exploit such materials for the development of optochemical nanosensors for a wide range of environmental monitoring applications, from chemical detection in gaseous or liquid phase $[6,49-53]$ to hydrogen detection at cryogenic temperatures suitable for aerospace applications [51]. In particular, the possibility of exploiting such materials in conjunction with the optical fiber technology could enable the development of optoelectronic noses and tongues capable of air and water quality monitoring, characterized by ppm and sub-ppm resolutions, good recovery features, and fast responses, as it will be seen in Section 4.

\section{OPTOCHEMICAL NANOSENSORS IN REFLECTOMETRIC CONFIGURATION: PRINCIPLES, FABRICATION, AND CHARACTERIZATION}

This section is focused on the description and operating principle of the optoelectronic sensing configuration adopted for the realization of the carbon nanotubes-based optochemical nanosensors (i.e., the reflectometric configuration), and the optoelectronic scheme for their robust, continuous, and real-time interrogation. The deposition technique exploited for the SWCNTs integration with the optical fiber technology, the molecular engineering Langmuir Blodgett (LB) method, is also presented and described in detail together with an extensive characterization of the structural and morphological properties of the fabricated chemosensitive coatings.

\subsection{Sensing configuration and operating principle}

The reflectometric configuration is essentially based on a low finesse and extrinsic Fabry-Perot (FP) interferometer and, as schematically shown in Figure 1, uses a thin sensitive film deposited at the distal end of a properly cut and prepared silica optical fiber (SOF) to produce an FP cavity. The thin film acts as an optical cavity where the first mirror is given by the fiber/sensitive layer interface whereas the second one is given by the sensitive layer/external medium interface.

First described in 1899 by Fabry and Perot [54], the interferometer known by their names makes use of multiple reflections between two closely spaced surfaces. In fact, the light is partially reflected each time it reaches the second surface, resulting in multiple offset beams which can interfere with each other (see Figure 2).

The amount of light reflected at the first interface can be calculated as the sum of the multiple reflected beams and is strongly influenced even by very small changes of the distance between the two surfaces (the sensitive layer thickness) or its optical properties (the sensitive layer refractive index) [55]. This explains the massive use of such configuration in fiber optic-based sensing in the two past decades, especially for the detection and measurements of various physical, chemical, and biomedical parameters [56, 57]. All these characteristics, combined with the possibility of integrating a number of sensitive materials with the optical fibers by means of very simple, low-cost, and versatile deposition techniques, make it one of the most attractive and useful optoelectronic configurations especially suitable for practical applications.

The principle of operation of an optoelectronic sensor in reflectometric configuration relies thus on the fact that a modulation of the intensity of light reflected at the fibersensing overlay interface occurs due to small changes of the layer thickness $\left(d_{\text {film }}\right)$ and its complex refractive index $\left(\tilde{n}_{\text {film }}\right)$. As a matter of fact, the fiber-film reflectance can be expressed as [58]

$$
R=\left|\frac{r_{12}+r_{23} \cdot e^{-i \cdot \tilde{k}_{\mathrm{flm}}}}{1+r_{12} \cdot r_{23} \cdot e^{-i \cdot \tilde{k}_{\mathrm{film}}}}\right|^{2}
$$

with

$$
\begin{aligned}
r_{12} & =\frac{n_{f}-\tilde{n}_{\mathrm{film}}}{n_{f}+\tilde{n}_{\mathrm{film}}}, \quad r_{23}=\frac{\tilde{n}_{\mathrm{film}}-n_{\mathrm{ext}}}{\tilde{n}_{\mathrm{film}}+n_{\mathrm{ext}}}, \\
\tilde{k}_{\mathrm{film}} & =\frac{2 \pi \cdot\left(2 \cdot \tilde{n}_{\mathrm{film}} \cdot d_{\text {film }}\right)}{\lambda} \\
& =\frac{4 \pi \cdot n \cdot d_{\text {film }}}{\lambda}-i \frac{4 \pi \cdot k \cdot d_{\text {film }}}{\lambda}=\beta_{\mathrm{film}}-i \alpha \cdot d_{\mathrm{film}},
\end{aligned}
$$

where $\tilde{n}_{\text {film }}=n-i \cdot k$ is the square root of the complex dielectric constant $\left(\tilde{\varepsilon}_{\text {film }}=\varepsilon_{r}-i \cdot \varepsilon_{i}\right)$ of the sensitive fiber coating, $\alpha=4 \pi \cdot k / \lambda$ is the overlay absorption coefficient, $n_{f}$ and $n_{\text {ext }}$ are the optical fiber and external medium refractive index, and $\lambda$ is the optical wavelength. Thus, the reflectance changes due to the chemical interaction between the sensing overlay and the target analyte can be expressed as follows:

$$
\begin{aligned}
\Delta R & =\left(\frac{\delta R}{\delta n}\right) \cdot \Delta n+\left(\frac{\delta R}{\delta k}\right) \cdot \Delta k+\left(\frac{\delta R}{\delta d_{\text {film }}}\right) \cdot \Delta d_{\text {film }} \\
& =S_{n} \cdot \Delta n+S_{k} \cdot \Delta k+S_{d} \cdot \Delta d_{\text {film }},
\end{aligned}
$$

where $S_{n}, S_{k}$, and $S_{d}$ are the sensitivities against the variations of the real and imaginary parts of the complex refractive index and against the overlay thickness, respectively. It is noteworthy that these three sensitivity factors $S_{n}, S_{k}$, and $S_{d}$ strongly depend upon the geometrical and electrooptical properties of the sensitive nanocoatings and upon the environmental condition (e.g., vapor or liquid phase), and for this reason they have to be properly considered case by case.

\subsection{Reflectometric sensor interrogation scheme}

So far, a variety of schemes have been proposed for the interrogation of a fiber optic sensor based on the FP cavity, the 


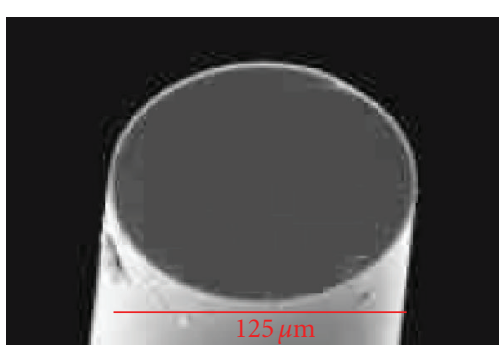

(a)

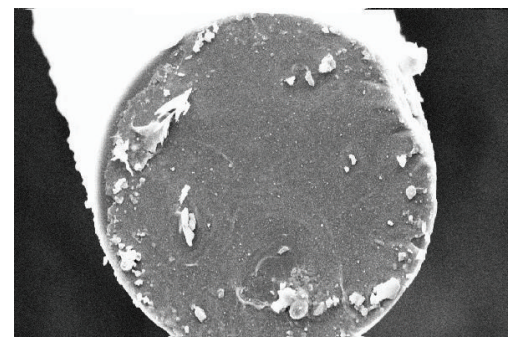

(b)

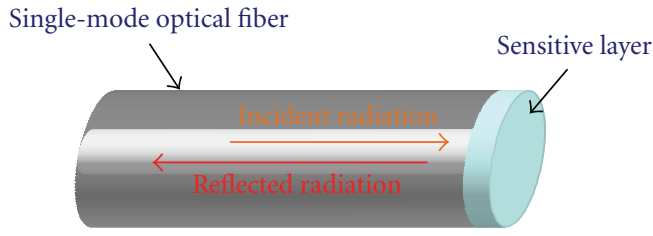

(c)

FIGURE 1: SEM images of (a) a properly cut bare fiber and (b) of a fiber tip coated by a thin sensitive layer; (c) schematic view of the Fabry-Perot-based configuration.

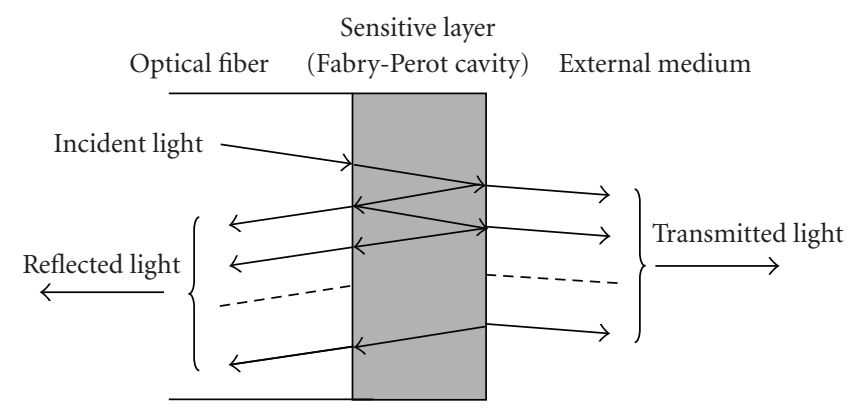

FIGURE 2: Schematic representation of the multiple reflected beams in a Fabry-Perot cavity.

most used ones relying on spectrum-modulating approach, and single wavelength reflectometry [59]. Here the attention has been focused on this last technique, which is simple to implement and requires just few widespread commercial and low-cost optoelectronic components while preserving excellent performances. In addition, it enables the fabrication of cost-effective, reliable, robust, and portable equipments, which are factors of crucial importance for in situ and longterm monitoring applications and for the desired technology transfer to the market. The typical interrogation scheme enabling the single wavelength reflectance monitoring of an FP cavity realized on the distal end of an optical fiber is shown in Figure 3(a).

The sensitive interface is lighted by a pigtailed superluminescent light emitting diode (SLED) operating in one of the two main telecommunication wavelengths $(1310 \mathrm{~nm}$ and $1550 \mathrm{~nm}$ ). A source controller is used to keep the SLED at a constant temperature and feed it with an input current, which allows the source to emit an optical power of few $\mathrm{mW}$. This power is split by a $2 \times 2$ in-fiber directional coupler, providing the necessary connections between light source, sensing interface, and two InGaAs photodiodes. The first photodiode is for the measurement of the reflected signal:

$$
V_{\text {signal }}=k_{1} \cdot Z_{1} \cdot P_{\text {source }} \cdot R,
$$

where $P_{\text {sourse }}$ is the power emitted by the source, $k_{1}$ is a constant which takes account of the power losses due to the coupling factor of the $2 \times 2$ directional coupler and the photodiode responsivity, $Z_{1}$ represents a gain factor introduced by the first photoreceiver device. The second arm of the $2 \times 2$ coupler is used to provide a valid intensity monitoring channel for the compensation of the sensor response. As a matter of fact, the second photodiode provides a voltage signal directly related to the optical power levels within the optical chain according to

$$
V_{\text {source }}=k_{2} \cdot Z_{2} \cdot P_{\text {source }}
$$

where $k_{2}\left(k_{2} \neq k_{1}\right)$ takes into account the losses coming from the connection between source, $2 \times 2$ coupler, and photodiode and $Z_{2}\left(Z_{2} \neq Z_{1}\right)$ is a gain factor introduced by the second photoreceiver device. The intensity compensation is obtained by considering the ratio between the voltage signals at the two photoreceivers according to

$$
I=\frac{V_{\text {signal }}}{V_{\text {source }}}=\frac{k_{1} \cdot P_{\text {source }} \cdot Z_{1} \cdot R}{k_{2} \cdot Z_{2} \cdot P_{\text {source }}}=\alpha \cdot R \text {, }
$$

where $\alpha=k_{1} \cdot Z_{1} / k_{2} \cdot Z_{2}$ takes into account all the setup parameters. As evident, the normalized response of the sensor is only sensitive to the change in the reflectance induced by chemical sorption within the sensing overlay and not to the fluctuations of the optical power levels along the whole measurement chain. In the followings, the relative change of the sensor output $\Delta I / I_{0}$ has been considered (where $I_{0}$ is the output signal in the reference or initial condition), which, in turn, corresponds to the relative reflectance change occurring at the fiber-sensitive layer interface $\left(\Delta R / R_{0}\right)$.

Synchronous detection is typically implemented to enhance the system performances, by amplitude modulating the light source at $500 \mathrm{~Hz}$ and retrieving the photodetector voltages by using a dual channel lock-in amplifier. In addition, a Time Division Multiplexing (TDM) approach is typically exploited to perform the quasisimultaneous interrogation of up to eight optical probes by means of a multichannel fiber optic switch. Finally, the optical data can be stored in a notebook controlling the sensing process in LabView software by a NI-DAQ card. The overall setup was properly engineered in order to provide robust, compact and portable equipment (see Figure 3(b)). 


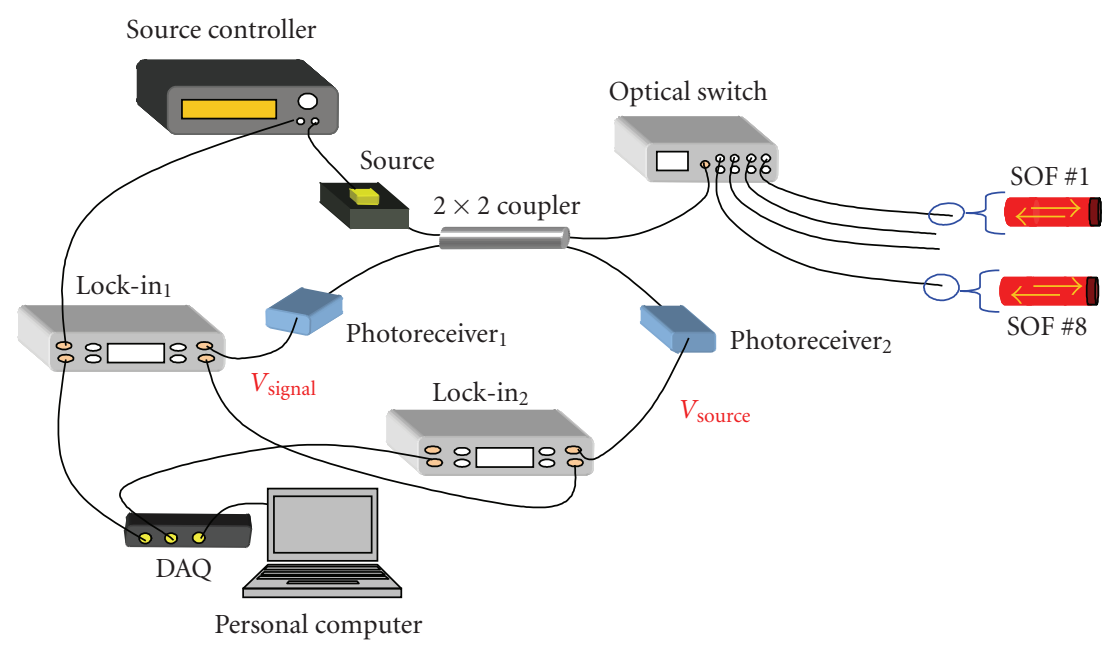

(a)

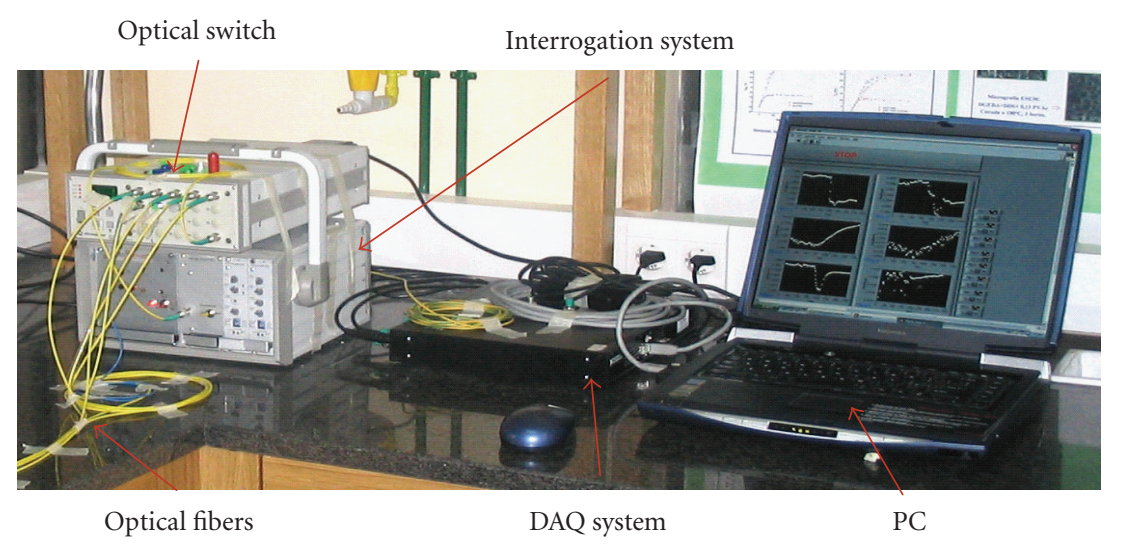

(b)

FIGURE 3: (a) Schematic illustration of the typical interrogation scheme adopted for the single wavelength reflectance monitoring of an optical cavity realized upon the fiber tip. (b) Image of the compact and portable equipment exploited for the interrogation of the FP-type optical fiber sensors.

\subsection{Optochemical nanosensor fabrication}

The realization of homogeneous thin films of SWCNTs with a controllable thickness is an important basis for the future development of their scientific understanding and technological applications. Proper manipulation techniques are required for applying thin films of carbon nanotubes on substrates that do not allow direct grow methods. Although various proposals exist for their incorporation into devices, in single tube or thin film architectures [60], here the LB technique has been chosen as a way to transfer nanometer-scale layers of SWCNTs upon either bare optical fibers or cadmium arachidate (CdA) buffer-linker material, previously deposited (by the same technique) upon the fiber end in order to improve the carbon nanotubes adhesion on the sensors surface. The CdA has been chosen as buffer material due to its peculiar amphiphilic molecular structure suitable for LB deposition process $[61,62]$.

The LB-technique is one of the most promising techniques for preparing such thin films as it enables the precise control of the monolayer thickness, homogeneous deposi- tion of the monolayer over large areas, and the possibility of making multilayer structures with varying layer composition [63]. An additional advantage of the LB technique is that monolayers can be transferred on almost any kind of solid substrate. However, these advantages have to be traded with the low speed of the deposition procedure as well as the limited number of materials suitable for this technique. As schematically represented in Figure 4, the molecules of the films to be deposited are firstly dispersed onto the surface of a subphase, typically oriented with the hydrophobic part upwards and with the hydrophilic one immersed in water. Subsequently, a reduction of the surface area occupied by each molecule is performed by means of moving barriers in order to produce a solid phase of a given surface pressure in which the molecules are densely packed forming a highly ordered array [64]. From this phase, the molecules can be transferred to a properly cleaned and prepared solid substrate by its dipping through the condensed Langmuir layer.

As the solid phase is reached only at high surface pressures, a continuous reduction of the moving barriers is performed when the molecules are transferred from the 

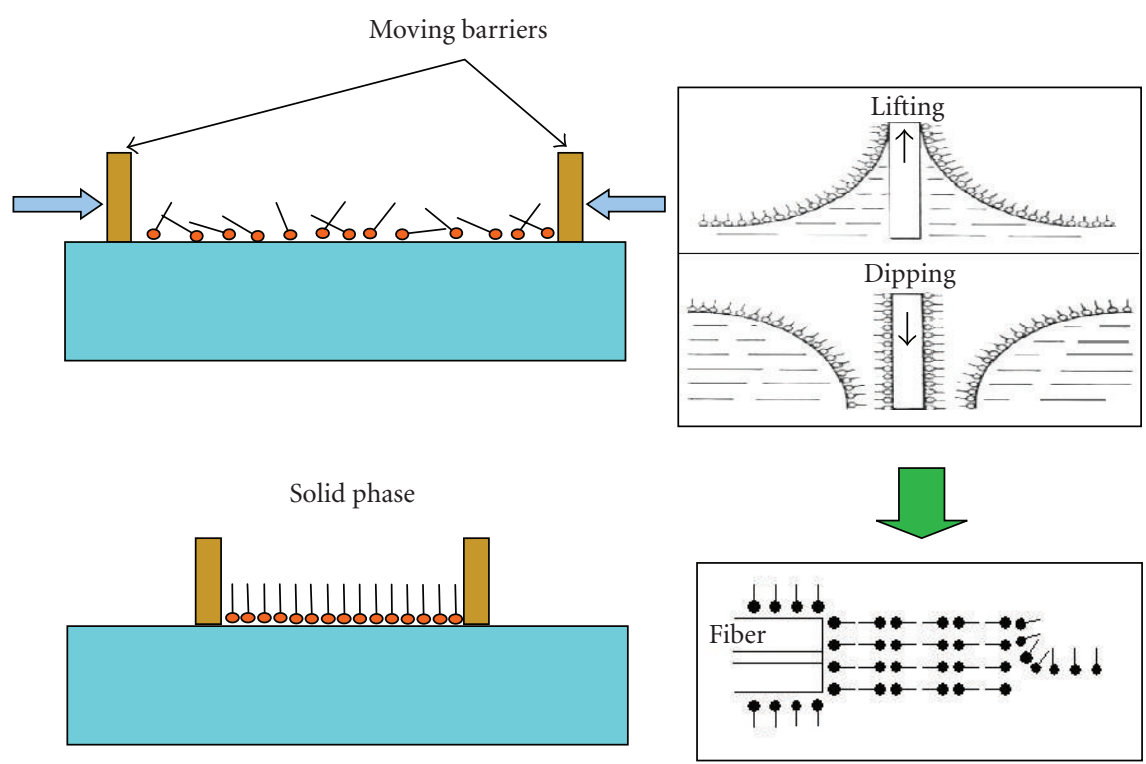

FIGURE 4: Schematic representation of the Langmuir-Blodgett deposition procedure.

subphase to the substrate in order to keep the surface pressure constant, ensuring that the solid phase is maintained. Repeated dipping of the same substrate is also possible, resulting in the deposition of a thin film with one monolayer at a time. The precise control and uniformity of film thickness in the submicron range allowed by the LB technique is essential especially for the optical interference principle used in the reflectometric configuration to convert refractive index changes into optical intensity changes.

For SWCNT film deposition, a solution $(0.2 \mathrm{mg} / \mathrm{mL})$ of SWCNT pristine material in chloroform has been spread onto a subphase constituted by deionized water $(18 \mathrm{M} \Omega)$ with $10^{-4} \mathrm{M}$ of $\mathrm{CdCl}_{2}$. The subphase $\mathrm{pH}$ and the temperature were 6.0 and $23^{\circ} \mathrm{C}$, respectively. The monolayer has been compressed with a barrier rate of $15 \mathrm{~mm} / \mathrm{min}$ up to a surface pressure of $45 \mathrm{mN} / \mathrm{m}$. The single layer has been deposited with a dipping rate of $3 \mathrm{~mm} / \mathrm{min}$ and the transfer ratio of the monolayers from the subphase to the surface of the optical fiber sensor substrate, either bare or already coated by 20 monolayers of CdA [50], was in the range of 0.5 to 0.7 . After a proper drying of 12 hours overnight, the sensing multilayers deposited were ready for the vapor testing. The SWCNT pristine material has been purchased from Carbon Nanotechnologies Inc. (Houston, Tex, USA), and contains primarily SWCNTs with a small amount (approx. 1\%) of amorphous carbon and $4-6 \%$ of catalyst Fe particles. The nanotube diameters are roughly Gaussian distributed and centered at about $2 \mathrm{~nm}$. The raw material of commercial nanotubes has been used as-bought, without any purification treatment. The samples were prepared by sonicating SWCNT suspension in chloroform for 1 hour at room temperature prior to the deposition. For the fabrication of the carbon nanotubes-based probes, before the LB deposition procedure the standard silica optical fibers have been previously accurately polished from the acrylic protection and cleaved with a precision cleaver in order to obtain a smooth and plane surface. Then, they have been washed in chloroform and dried with gaseous nitrogen to be ready for the CdA or SWCNT deposition. The number of CdA and/or SWCNT monolayers deposited onto the sensor surface have been controlled by simply choosing how many times the substrate is dipped inside or lifted from the solution containing the monolayer to transfer.

\subsection{Sensor characterization: structural and morphological features of SWCNT overlays}

The rational design of a chemical sensor and of its performances is something which is possible only if the sensitive material properties and the way they are affected by different deposition parameters or ambient conditions are well known and understood. To this aim an extensive characterization of the as-bought SWCNT powders as well as of the deposited LB SWCNT films has been carried out in order to investigate their structural and morphological features. Such characterization involved X-ray diffraction (XRD) and Raman spectroscopy analyses, high-resolution transmission electron microscopy (HRTEM), and scanning electron microscopy (SEM) observations. In particular, Figure 5 reports the typical XRD spectrum of as-bought SWCNT powder $(1 \mathrm{mg})$ material. The pattern exhibits a well-defined graphite-like (002) diffraction peak at $2 \theta=$ $26.5^{\circ}$, and a broad band centered at lower $2 \theta=22^{\circ}$ from amorphous carbon or nonnanotube carbon material.

Further XRD analyses performed on CdA multilayers deposited on glass substrates evidenced a CdA monolayer spacing of about $2.8 \mathrm{~nm}$ for cadmium arachidate. The same measurements, performed with an SWCNT multilayer, revealed a carbon nanotubes monolayer spacing of about $2.0 \mathrm{~nm}[50]$.

The HRTEM images of an SWCNT powder, reported in Figure 6 at (a) low and (b) high magnifications, confirm the 


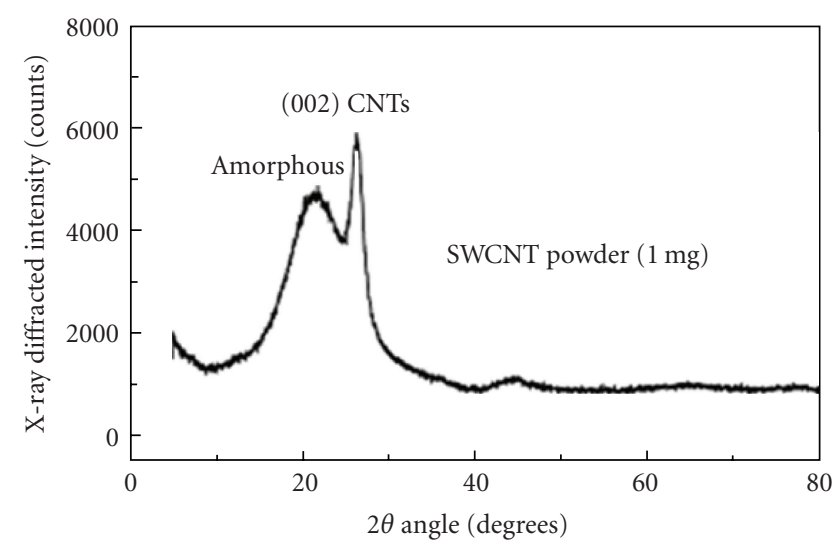

FIGURE 5: X-ray diffraction spectrum obtained from HiPco SWCNT powder (1 mg).

nanometric dimension of the carbon tubes and reveal the presence between them of some Fe metal particles, a typical catalyst used in the HiPco production process of carbon nanotubes. Generally, the SWCNT powders contain not only carbon tube nanostructures but also carbonaceous particles such as amorphous carbon, fullerenes, and transition metals introduced as a catalyst during the synthesis. $[65,66]$.

These impurities sometimes hinder the fine characteristics of the carbon nanotubes and limit the best performance of the SWCNTs-based functional devices. For this reason, effective purifications for the removal of the by-products (amorphous carbon, fullerenes, catalyst metal particles) of SWCNT synthesis are needed in order to fully exploit their outstanding properties. Finally, Figure 6(c) reports the typical SEM images of CdA-buffered LB SWCNT films deposited upon an SOF tip. It demonstrates the success of the integration of carbon nanotubes with the optical fiber and reveals their attitude to adhere one to each other forming bundles or ropes with a spaghetti-like arrangement. In the investigated cases, such CNT mats are characterized by mean diameter and length which have been estimated to be in the range $5-40 \mathrm{~nm}$ and $1-15 \mu \mathrm{m}$, respectively.

Also Raman spectroscopy analyses have been conducted to characterize the fabricated LB films based on SWCNTs already deposited upon the optical fiber tip. To this aim, a Raman microscope functioning in backscattering configuration employing an HeNe laser $(633 \mathrm{~nm})$ and $50 \mathrm{x}$ and $100 \mathrm{x}$ objective lenses was used. The results are shown in Figure 7, where the typical Raman spectrum of SWCNTs has been reported.

They confirm the single-walled and semiconducting nature of the CNTs [67] as well as their integration with the optical fiber transducers. The characteristic multipeak feature "G-band" at about $1580 \mathrm{~cm}^{-1}$, corresponding to carbon atom vibration tangentially with respect to the nanotube walls, together with the less remarkable disorder-induced "D-band" peak typically in the range $1300-1400 \mathrm{~cm}^{-1}$, representing the degree of defects or dangling bonds [67], can be easily revealed. The large ratio of $G$ to $D$ peaks here observed gives us an indication of an ordered structure of the deposited SWCNT overlay, whereas the observation of the two most intense $\mathrm{G}$ peaks (labeled $\mathrm{G}+$ and $\mathrm{G}-$ ) confirms the absence of MWCNTs.

\section{ENVIRONMENTAL MONITORING APPLICATIONS: EXPERIMENTAL RESULTS}

This section provides a review of the results obtained during the last few years of research focused on the development of SWCNTs-coated optochemical nanosensors for numerous environmental monitoring applications. In particular, in the following sections VOC detection in air, chemical trace monitoring in water, and $\mathrm{H}_{2}$ detection at cryogenic temperature suitable for aerospace applications will be presented. The results here reported reveal the potentiality of the SWCNTs-coated chemosensors to be employed as very promising optochemical devices for applications related to the environmental monitoring either in air or in liquid phase, at room temperature and likewise at a temperature as low as $113 \mathrm{~K}$.

\subsection{Room temperature detection of VOCs in air}

The investigation and characterization of the VOC detection performance of SWCNTs-coated optochemical nanosensors have been carried out by means of an experimental setup ad hoc designed and realized, a schematic representation of which is reported in Figure 8. In particular, the optical fiber probes have been located in a properly designed test chamber realized in stainless steel. The volume of the test chamber was $1200 \mathrm{~mL}$, the total flow rate per exposure has been kept constant at $1000 \mathrm{~mL} / \mathrm{min}$ and the vapors have been generated by the bubbling method. The gas flow rate has been controlled by a mass flow-meter driven by a controllerunit communicating with a PC via standard RS-485 serial bus. The controller unit was able to drive up to eight different gas-channels, and the gas flow rate in each gas channel was regulated by a dedicated mass flow meter with a full scale of the mass flow ranging from 10 to $1000 \mathrm{~mL} / \mathrm{min}$. Numerous tests have been performed by using nitrogen or dry air as reference and carrier gas to transport the generated vapors inside the test ambient: dry air has been chosen because of the higher stability demonstrated by the optical fiber sensor signals. All the experimental measurements have been conducted at room temperature.

The capability of SWCNT overlays of undergoing changes in their complex refractive index and thickness as consequence of the adsorption of target analyte molecules has been demonstrated for the first time in 2004 [49]. In that case, LB films consisting of SWCNT bundles were transferred upon the optical fiber tip by using a buffer LB multilayer of CdA predeposited on the sensor surface in order to promote the adhesion of the CNTs. The fabricated probes were exploited for the detection of several VOCs, such as isopropanol, methanol, ethanol, toluene, and xylene. However, in 2005, multilayers of SWCNTs with different thicknesses were successfully deposited directly upon the optical fiber surface by a modification of the LB process [68], resulting in an improvement of 


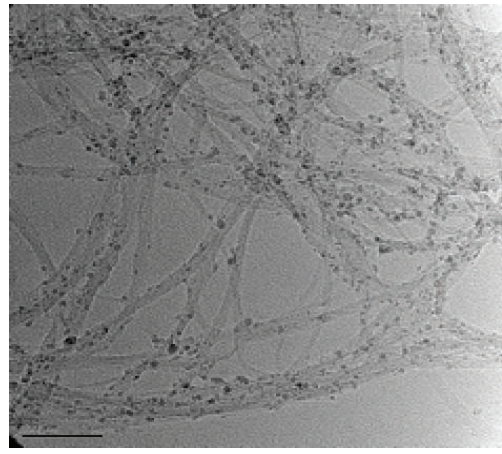

(a)

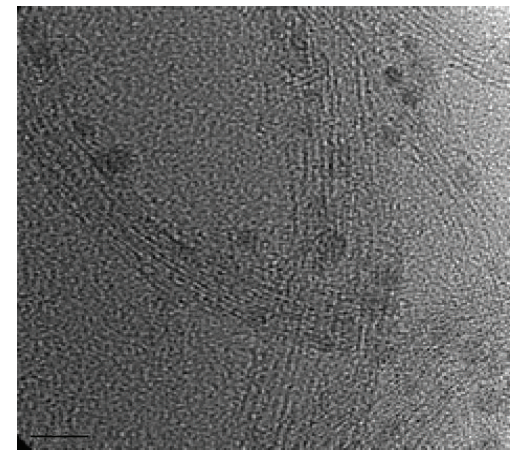

(b)

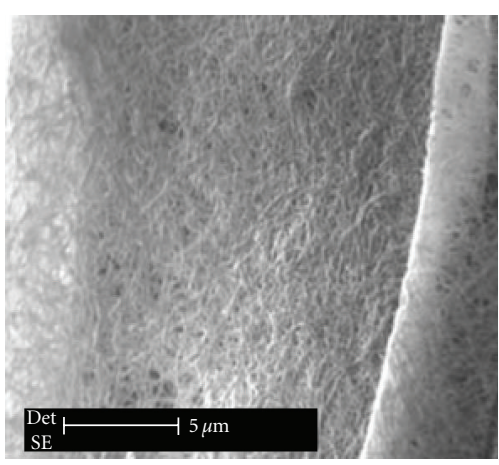

(c)

FIGURE 6: (a), (b) High resolution TEM images of SWCNT powder at (a) low and (b) high magnifications, (c) SEM images of CdA-buffered SWCNT LB films deposited upon an SOF sensor substrate.

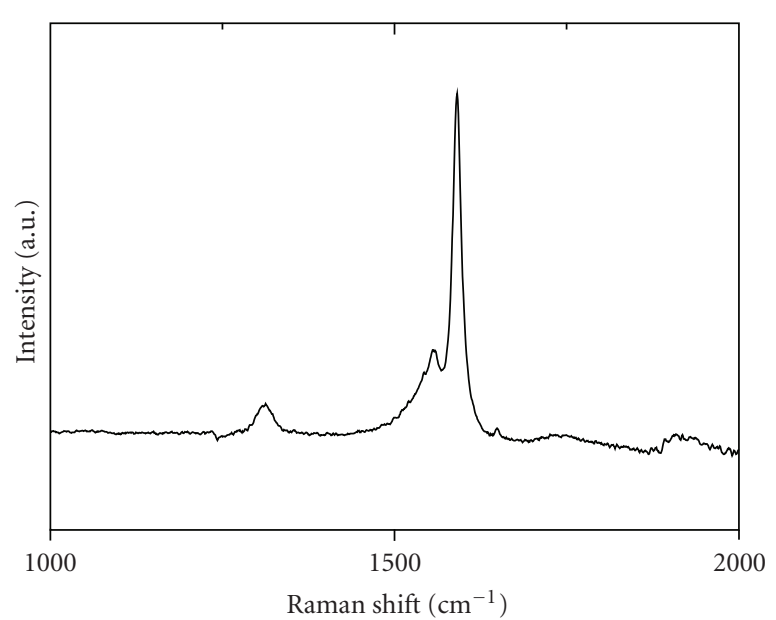

FIGURE 7: Typical Raman spectrum of an LB SWCNT film directly deposited upon the optical fiber tip.

the sensing performance of the unbuffered configuration with respect to the buffered case, especially in terms of sensor sensitivity. As an example, Figure 9 shows the highest relative reflectance changes $\left(\Delta R / R_{0}\right)$ exhibited by a fiber optic chemosensor coated with a CNTs film composed of four SWCNT monolayers (namely, SOF-4) when exposed to 30-minute decreasing concentration pulses of xylene vapors with respect to the counterpart optoelectronic sensor arranged in the CdA buffered configuration (2 monolayers of SWCNTs deposited onto 20 monolayers of CdA). In both cases, significant reflectance changes occurred upon target analyte exposure as a consequence of the variation in the SWCNT overlay refractive index promoted by the toluene molecules adsorption inside the sensitive material. Both sensors exhibited the capability of detecting traces of the chemical under investigation at few tens of ppm levels combined with a fast response, a complete reversibility of the sensor responses (which grants the reuse of the sensor after a given measurement), and a marked dependence of the response times upon the analyte concentration.
However, the sensor SOF-4 provided response changes more than one order of magnitude higher than the ones provided by the counterpart probe in the CdA-buffered configuration. In light of these results, strong interest was devoted to the investigation of the sensing capabilities of SWCNTs-based optochemical sensors arranged in the unbuffered configuration against several VOCs at room temperature.

Figure 10(a) reports the results of the toluene vapor testing carried out by exposing at room temperature the transducers SOF-4 to four pulses of toluene with concentration in the range $54-93 \mathrm{ppm}$. The results obtained confirmed the behavior of the fiber optic nanosensor exhibited during the xylene testing: as a matter of fact, also in this case an optical reflectance increase on exposure was observed as a consequence of the analyte molecule adsorption. A less pronounced dependence of the response time on the toluene concentration was noticed, revealing that different adsorption dynamics occur depending on the VOC under investigation, as it will be examined more in detail in Section 4.1.2. Also, a slight drift in the signal baseline can be recognized, due to little thermal changes in the not perfectly thermostated test chamber. After 5 hours of vapors testing, the total $\Delta R / R_{0}$ due to a temperature drift of $2.4^{\circ} \mathrm{C}$ was approximately $2.8 \cdot 10^{-3}$. Furthermore, in order to investigate the reliability of the proposed transducers, a repeatability test has been carried out for the same optoelectronic nanosensor.

The results are shown in Figure 10(b), where the reflectance changes that occurred as a consequence of two exposures at $21 \mathrm{ppm}$ of xylene, at room temperature, have been reported. The exposure time has again been kept constant at 30 minutes. As evident, the optochemical probe is demonstrated to be highly repeatable and reliable also at very low analyte concentrations. The results of the repeatability test have to be considered at constant temperature, because no thermal variation occurred during it. However, it is evident that the monitoring of thermal drifts and the compensation of their effects on the sensor response are strictly required in order to not reduce the system performance. The temperature monitoring could be implemented by means of appropriate Fiber Bragg Grating temperature sensors, which 


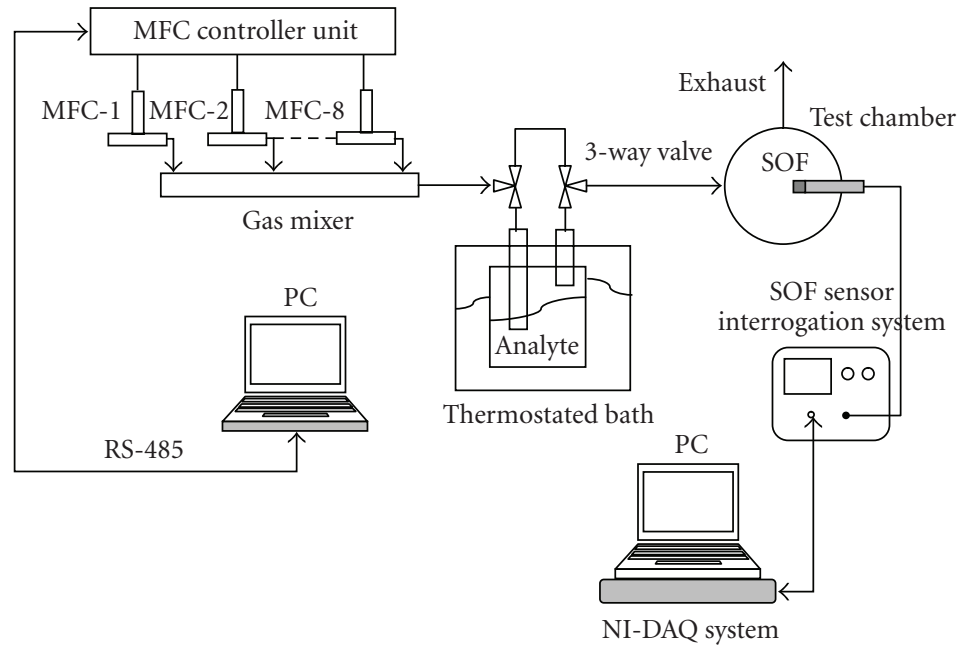

(a)

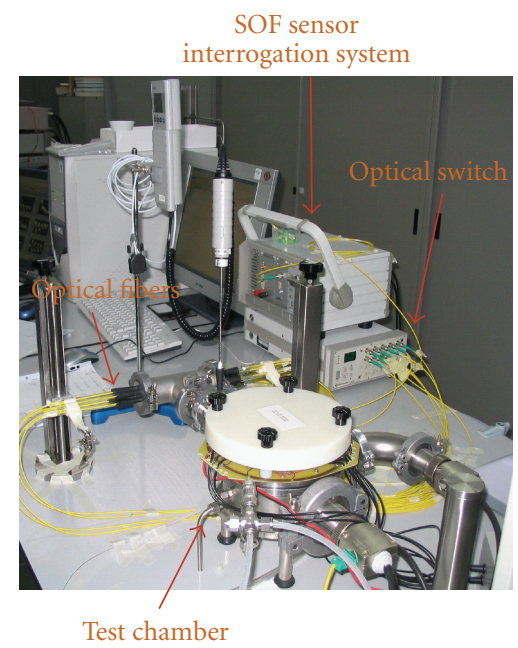

(b)

FIGURE 8: (a) Schematic representation and (b) photogram of the experimental setup used for the vapor testing.

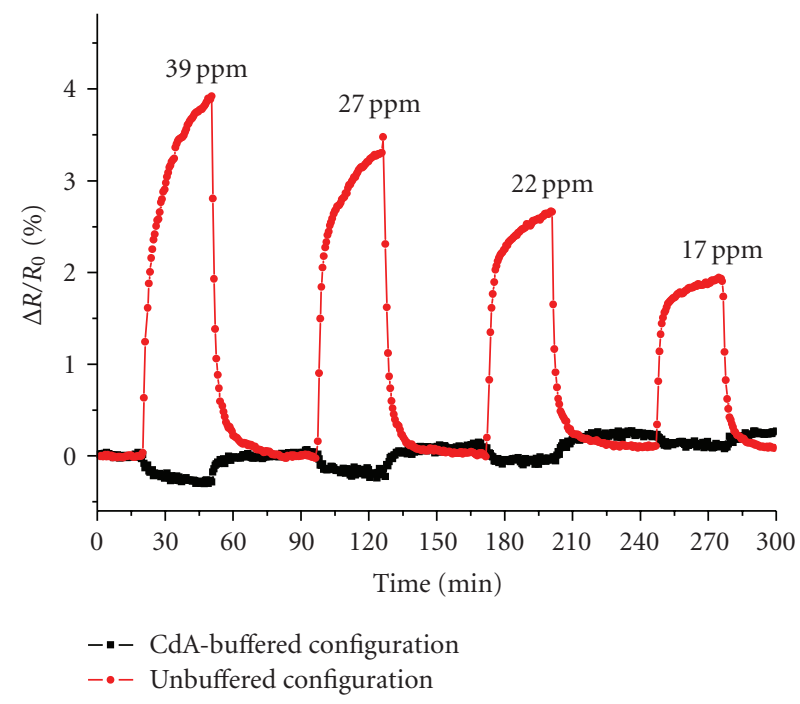

Figure 9: $\Delta R / R_{0}$ occurred on xylene vapor exposure to CdAbuffered and unbuffered SWCNTs-based optochemical nanosensors, at room temperature.

could be separately inserted in the test ambient or even integrated with the optical fiber probe [69]. Similarly, the SWCNTs-based optoelectronic chemosensors demonstrated a relevant sensitivity also to humidity changes within the test environment [68]. As a matter of fact, controlled humidity test performed using an SOF sensor coated by two monolayers of SWCNTs onto 20 monolayers of CdA revealed a sensitivity as high as $-5.4 \cdot 10^{-4}$ for $1 \%$ humidity change, thus requiring a proper calibration and compensation of the sensors response, especially when high accuracy is necessary.

Figure 11 reports the calibration curves of the nanosensor SOF-4 obtained against toluene and xylene vapors at room temperature, which revealed an almost linear behavior in the investigated concentration ranges. In addition, the sensor sensitivities against both analytes, calculated as the relative reflectance change upon concentration unit $\left(S_{\text {analyte }}=\Delta R / R_{0} / C\right)[70]$, evidenced a higher response to xylene $\left(S_{\text {xylene }}=1.1 \cdot 10^{-3} \mathrm{ppm}^{-1}\right)$ than to toluene $\left(S_{\text {toluene }}=\right.$ $\left.4.7 \cdot 10^{-4} \mathrm{ppm}^{-1}\right)$. As a matter of fact, the SOF sensitivity to xylene is more than two times higher than the one to toluene, as evidenced by the ratio between them $\left(S_{\text {xylene }}^{\text {sof }} / S_{\text {toluene }}^{\text {soo }}=\right.$ 2.4).

By considering the minimum detectable values achievable with the exploited optical interrogation device $\left(\Delta R / R_{0 \min }=1.34 \cdot 10^{-4}\right)$, resolutions of approximately $290 \mathrm{ppb}$ and $120 \mathrm{ppb}$ have been estimated for toluene and xylene, respectively. These excellent resolutions are more than three orders of magnitude higher than those obtained by means of a fluorosiloxane polymer-based surface plasmon resonance (SPR) optical fiber sensor $(900 \mathrm{ppm}$ and $190 \mathrm{ppm}$, resp., for toluene and xylene) [71], and more than two orders of magnitude higher than that provided by a multimodal optical fiber sensor sensitized by phenyl-modified porous silica (100 ppm and $20 \mathrm{ppm}$, resp.) [72].

\subsubsection{Effects of the number of SWCNT monolayers and $\mathrm{CdA}$ buffer on the sensor sensitivities}

In the present section, the influence of the number of SWCNTs monolayers deposited upon the optical fiber end-face on the sensor performance will be discussed. The differences between the performance of the buffered and unbuffered configurations are also better discussed. To achieve this aim, four optochemical sensors coated by a different number of SWCNTs, directly deposited onto the bare substrates and also buffered by $20 \mathrm{LB}$ monolayers of CdA, have been simultaneously exposed to toluene and xylene vapors.

Figure 12(a) reports the relative reflectance changes versus the toluene concentration for all the tested nanosensors, at room temperature. It can be seen that an increase of the sensitivity has been obtained by passing from 


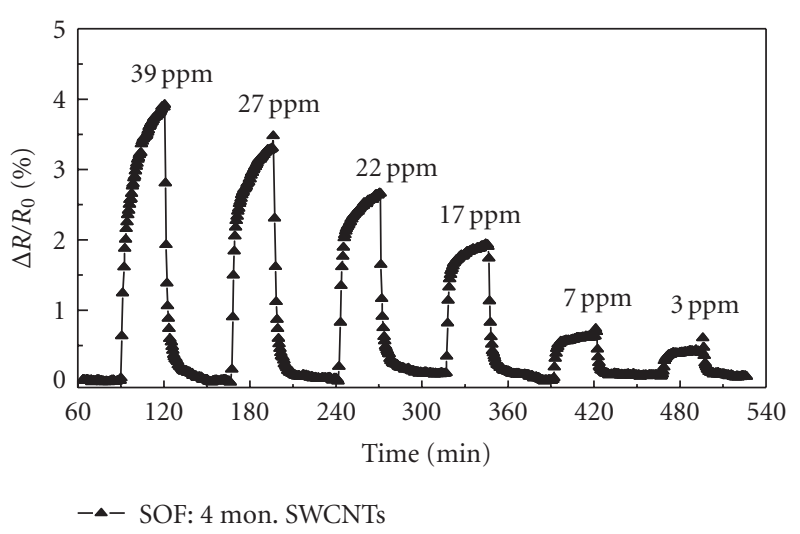

(a)

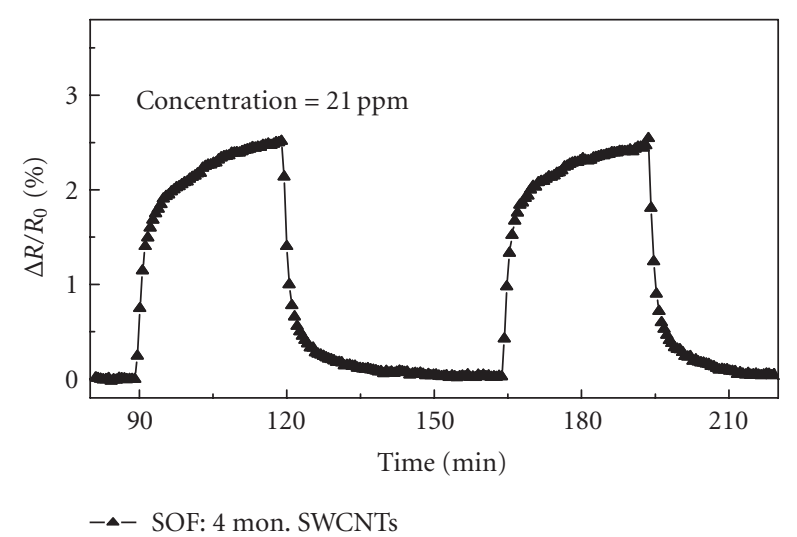

(b)

FIGURE 10: (a) Time responses of the sensor SOF-4, exposed to four decreasing concentration pulses of toluene vapors, at room temperature; (b) repeatability test, carried out by exposing the same sensor to 30-minute pulses of xylene vapors with a concentration as low as 21 ppm.

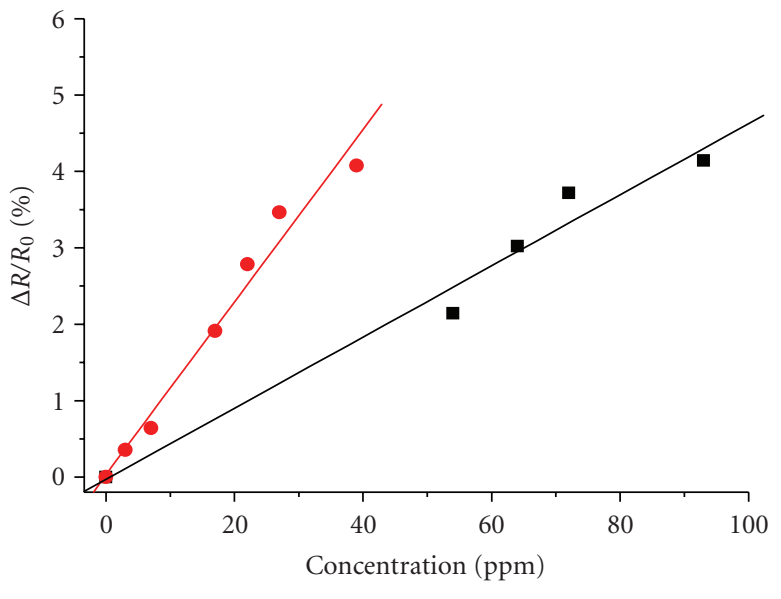

- SOF: toluene

- SOF: xylene

FIgURE 11: Calibration curves of the sensor SOF-4, exposed at room temperature to toluene and xylene vapors.

two $\left(0.9 \cdot 10^{-4} \mathrm{ppm}^{-1}\right)$ to four monolayers of SWCNTs $\left(4.7 \cdot 10^{-4} \mathrm{ppm}^{-1}\right)$ directly deposited onto bare SOFs (see Figure 12(b)). On the contrary, the optical chemosensors coated by a higher number of carbon nanotubes monolayers (twelve), and thus by a thicker SWCNT film, exhibited a negative sensitivity to toluene $\left(-0.7 \cdot 10^{-4} \mathrm{ppm}^{-1}\right)$. This means that for this sensor the fiber-film interface reflectance decreases on exposure. This is due to the fact that the film reflectance, and thus also the sensor sensitivity, is strongly dependent upon the thickness and refractive index of the CNT ovelay, in accordance with (1). As a matter of fact, depending upon the geometric features of the film deposited onto the optical fiber end, a sensor can exhibit either a positive or a negative sensitivity to a target analyte.

For the same reason, the CdA buffer multilayer, whose optical properties are quite similar to those of the standard optical fibers, and thus not optimized for the particular con- figuration exploited, dramatically decreases the sensitivity of optical fiber sensors $\left(-0.4 \cdot 10^{-4} \mathrm{ppm}^{-1}\right)$. It is noteworthy, however, that by choosing a buffer-linker material whose optical and geometrical features (such as the multilayer thickness and refractive index) are well optimized for the specific configuration, one could be able to strongly enhance the SOF sensor performance. From Figure 13, it can be seen that the four fiber optic chemosensors exhibited the same behaviors also in case of xylene vapor testing. Hence, it demonstrates that the inversion of the optoelectronic sensor sensitivities is not due to the particular analyte tested, but to the optical configuration exploited. This feature could be very useful for pattern recognition analysis in case of multitransducer approaches, where complementary sensors are exploited to improve the analyte discrimination.

\subsubsection{Response and recovery time analysis}

The experimental results so far reported evidenced a dependence of the sensor response times upon the analyte concentration. For this reason, a detailed analysis of the adsorption dynamic features of the proposed chemosensors towards both the tested hydrocarbons is required. To this aim, in this section an investigation of the response and recovery times of the sensor SOF-4, exposed to decreasing concentration pulses of toluene and xylene vapors, at room temperature, is reported. The exposure time was 30 minutes for each pulse. The response (recovery) time has been calculated as the time needed for the output signal to pass from $10 \%$ to $90 \%$ (from $90 \%$ to $10 \%$ ) of the total signal variation occurring on vapor exposure (recovery). The results obtained in the case of toluene are shown in Figure 14(a), and reveal a response time that increases with the analyte concentration. The minimum response time, obtained for 54 toluene ppm, was approximately 7 minutes while the maximum one, obtained for $93 \mathrm{ppm}$, was approximately 11 minutes. In addition, a quite constant recovery time as low as approximately 5 minutes has been estimated for the same transducer. 


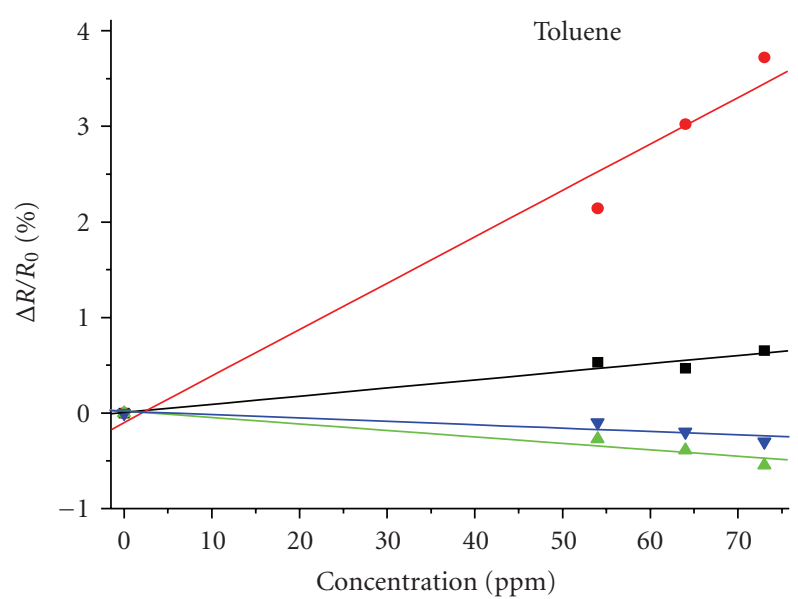

- SOF: 2 mon. SWCNTs

- SOF: 4 mon. SWCNTs

(a)
- SOF: 12 mon. SWCNTs

v SOF: $20 \mathrm{CdA}+10$ SWCNTs

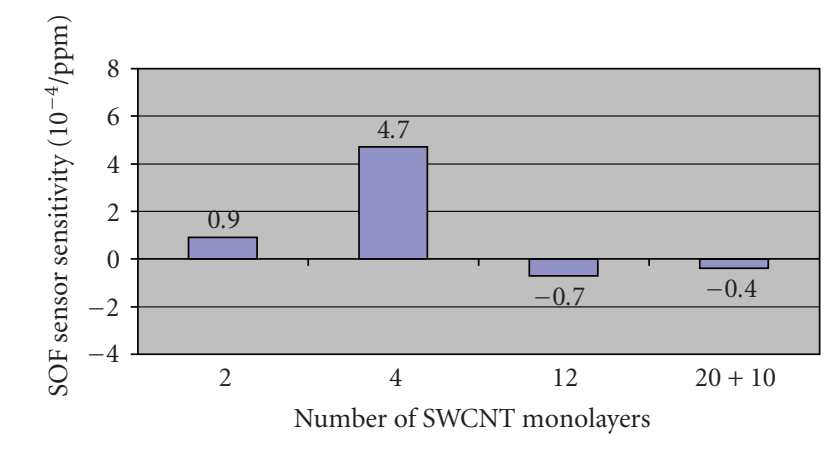

$\square$ Toluene

(1)

FIGURE 12: Comparison between the (a) calibration curves and (b) sensitivities obtained by exposing four fiber optic chemosensors coated by a different number of SWCNT monolayers to toluene vapors, at room temperature.

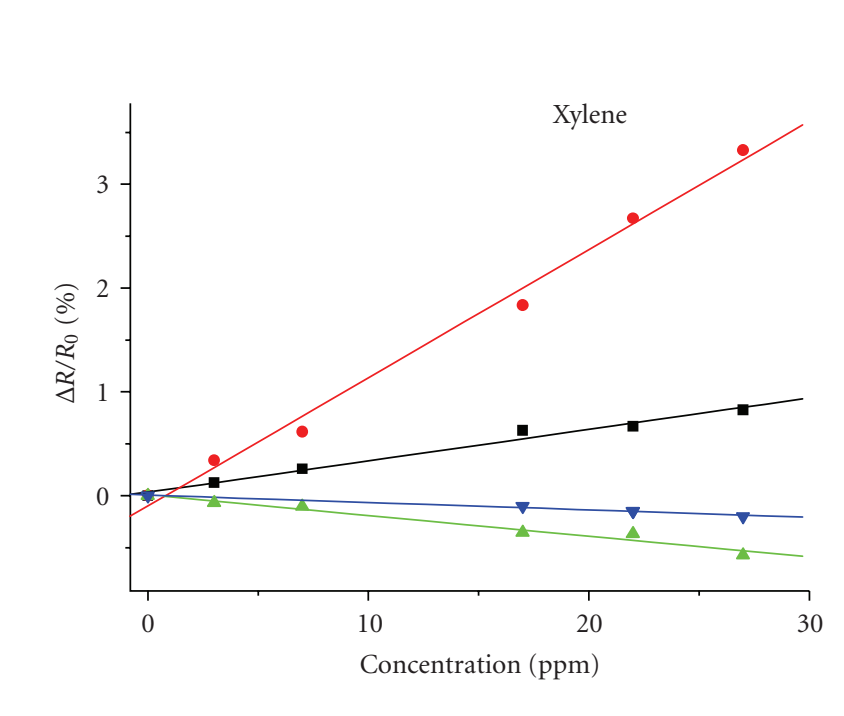

- SOF: 2 mon. SWCNTs

- SOF: 4 mon. SWCNTs
- SOF: 12 mon. SWCNTs

- SOF: $20 \mathrm{CdA}+10$ SWCNTs

\section{(b)}

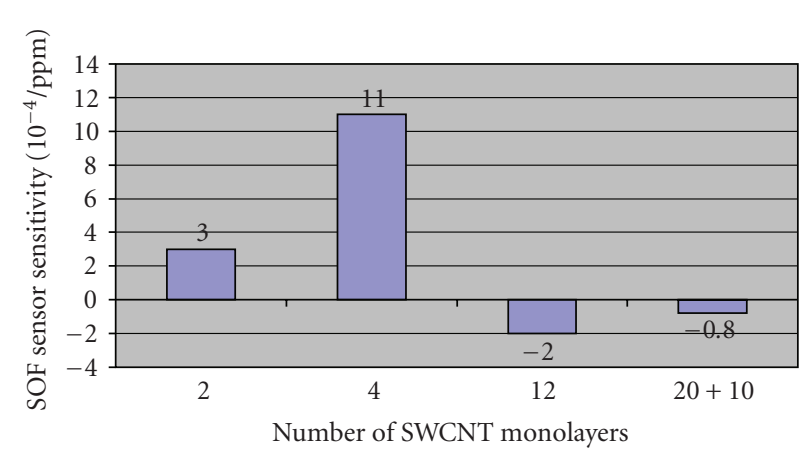

Xylene

(a)

FIGURE 13: Comparison between the (a) calibration curves and (b) sensitivities obtained by exposing four fiber optic chemosensors coated by a different number of SWCNT monolayers to xylene vapors, at room temperature.

Similar results have been observed in case of xylene exposures (see Figure 14(b)), with the SOF response times increasing from approximately 4 minutes $(3 \mathrm{ppm})$ to approximately 18 minutes ( $39 \mathrm{ppm})$. In addition, the recovery times of both sensors are quite constant as a function of the xylene concentration, with their values estimated to be of approximately 6 minutes. By comparing the response and recovery times towards the two tested VOCs, it turned out that the dynamic responses of the sensor SOF-4 are slower in the case of xylene vapors than in the case of toluene ones. This effect could be attributed to a different adsorption kinetics of the two target analyte molecules inside the CNT sensitive nanocoatings. It is noteworthy, however, that the response times of the proposed fiber optic chemosensors are relatively good taking into account the volume of the test chamber and the total flow rate per exposure.

It is also important noting that these times could be improved by depositing a lower amount of carbon nanotubes upon the sensor substrates, for example by decreasing the number of SWCNT monolayers. However, this could reduce the sensor sensitivities. As a matter of fact, Figure 15 reports a bar plot showing the mean response and recovery times of the sensors SOF-2 and SOF-4, obtained for a 17 ppm xylene vapors exposure, at room temperature. It can be clearly 


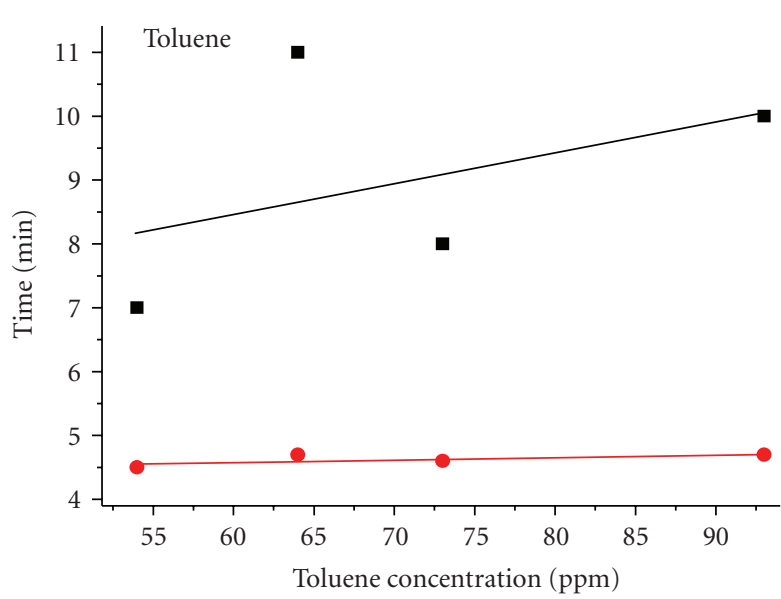

- SOF_4NTs: response time

- SOF_4NTs: recovery time

(a)

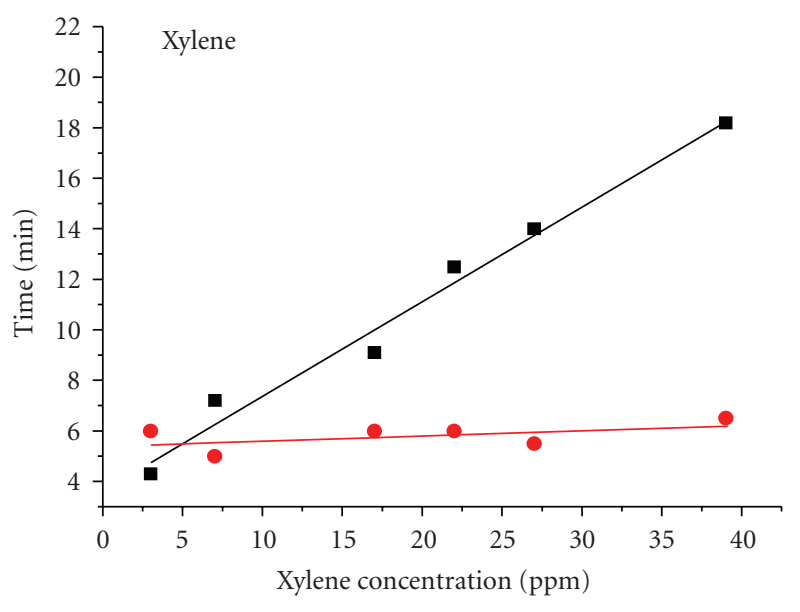

- SOF_4NTs: response time

- SOF_4NTs: recovery time

FIGURE 14: Response and recovery times obtained by exposing the sensor SOF-4 to (a) toluene and (b) xylene vapors, at room temperature.

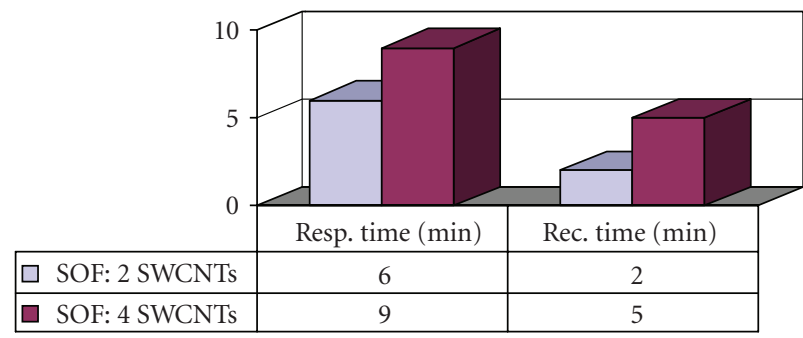

FIGURE 15: Comparison between mean response and recovery times of the fiber optic chemosensors SOF-2 and SOF-4, obtained for a 17 xylene ppm exposure, at room temperature.

observed that, even if a decrease of the sensor mean response time (from 9 to 6 minutes) as well as of the mean recovery time (from 5 to 2 minutes) can be achieved by reducing the SWCNT layer thickness, this results in a strong diminution of the sensor sensitivities. In fact, as observed in Section 4.1.1, a reduction from $1.1 \cdot 10^{-3} \mathrm{ppm}^{-1}$ to $3 \cdot 10^{-4} \mathrm{ppm}^{-1}$ has been observed by passing from the sensor SOF-4 to SOF-2. This means that the choice of the geometric features of the sensitive overlay has to be made by considering the tradeoff that exists between sensor sensitivity and response (and recovery) time.

\subsection{Chemical trace detection in water}

In this section, the attention has been focused on the feasibility of exploiting the excellent sensing properties of CNTs for the development of high-performance optoelectronic sensors capable of chemical trace detection in aqueous environment, at room temperature.

A schematic view of the experimental setup exploited for the chemical trace detection in water is reported in Figure 16. The SWCNTs-based optochemical transducers in

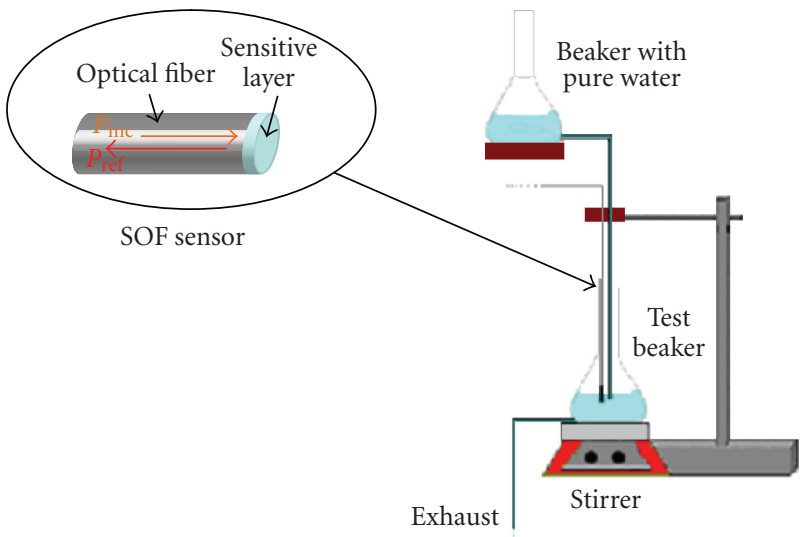

FIGURE 16: Schematic view of the experimental setup exploited for the chemical trace detection in water.

reflectometric configuration have been inserted in a Pyrex beaker containing pure water. The presence within the test ambient of the analyte under investigation has been promoted by its injection inside the beaker. The injected volume has been chosen, each time, in order to obtain the desired analyte concentration. The polluted water has been continuously stirred to ensure the maximum dispersion of the analyte. In addition, after each analyte exposure, the capabilities of the SOF sensors to recover the initial steady state level have been investigated by restoring the initial condition of pure water. To this aim, pure water has been continuously injected in the test chamber, while the contaminated water, previously present in it, contemporarily stilled out.

Before performing the toluene detection measurements, the stability within an aqueous environment of the SWCNT sensitive layers, deposited upon the fiber optic end-face, has been investigated. To the aim, a chemooptic sensor coated by 


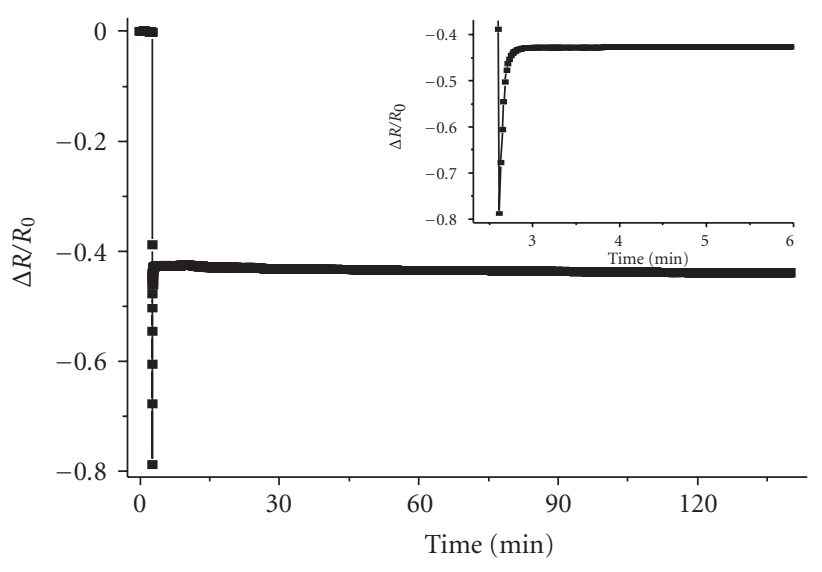

FIgURE 17: $\Delta R / R_{0}$ of the SWCNTs-based sensor occurred as a consequence of its insertion in water.

twelve monolayers of SWCNTs, whose sensing performance against toluene in air was already tested [6], has been successively inserted in a beaker containing pure water and also left for several days inside it. Figure 17 reports the $\Delta R / R_{0}$ of the optical probe occurred in correspondence of its insertion in water. It can be seen that, as it was inserted in water, a strong and fast reflectance decrease occurred as a consequence of the change in the external medium refractive index. This effect is followed by a slow variation of the reflectance itself (see the inset in Figure 17) until the equilibrium value is reached, which can be attributed to the interaction between the sensitive layer and the water molecules. In addition, once reached the equilibrium, the reflectance signal is stable, demonstrating that no degradations of the SWCNT sensitive layer occurred.

Once the carbon nanotubes stability has been verified, the same SOF sensor has also been tested for the room temperature detection of toluene in water. The analyte concentration range was $20-80 \mathrm{ppm}$ (calculated as $\mu \mathrm{L} / \mathrm{L}$ ). The results obtained are shown in Figure 18, where the $\Delta R / R_{0}$ that have occurred upon the injections of increasing concentrations of toluene have been reported. It can be observed that in correspondence of each toluene injection the interaction between analyte molecules and sensitive overlay results in a significant decrease of the reflectance. In addition, the CNTs-based SOF sensor provides complete recoveries of the steady-state value of uncontaminated water, which demonstrates its excellent desorption features. The slight drift observed in the sensor baseline can be attributed to thermal changes since the test chamber is not perfectly thermostated. However, this aspect, as already evidenced in the previous sections, can be easily addressed by using simple procedures to compensate the effects of temperature variations on the sensor response.

An estimation of the influence of noise on the sensor response has also been provided by calculating the noise effect on the sensor output $I$ in the steady state levels corresponding to the absence of analyte within the chamber. By considering the steady-state level in the worst case (maximum scattering in the sensor level) over a time interval

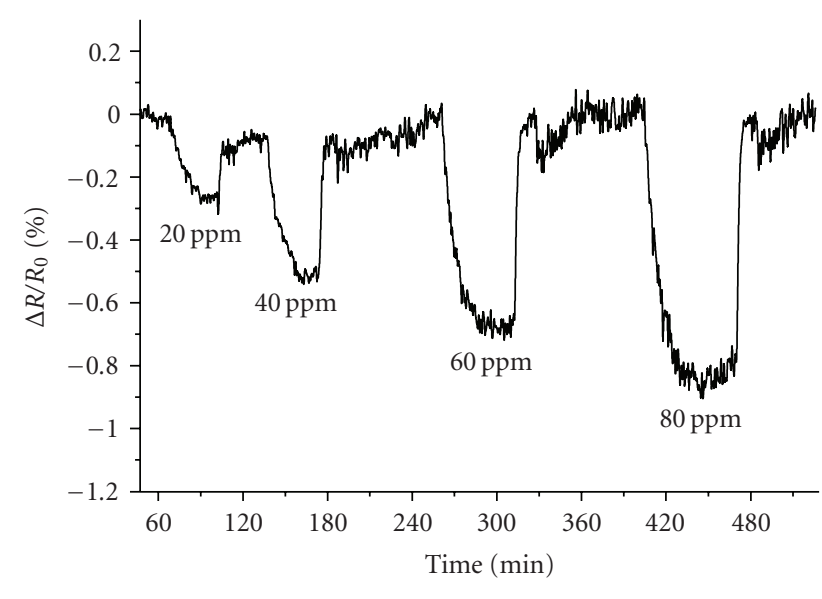

12 monolayers of SWCNTs

Figure 18: Time responses of the SOF sensor coated by 12 monolayers of SWCNTs, exposed to increasing concentrations of toluene, at room temperature.

of 15 minutes, a standard deviation $\sigma_{N}$ from the mean value of the sensor output $I_{0}\left(I_{0} \sim 0.9424\right)$ of approximately $6 \cdot 10^{-4}$ can be appreciated. By these results, a minimum detectable change of the sensor output $\Delta I / I_{0 \mathrm{~min}}$ (and thus a $\Delta R / R_{0 \mathrm{~min}}$ ) of $6 \cdot 10^{-4}$ has been estimated.

In Figure 19(a), the sensing performance of the SOF sensor coated by twelve monolayers of SWCNTs against toluene in air and aqueous environments has been compared.

Here, the reflectance changes that occurred in the two cases have been plotted versus the toluene concentration. As can be observed, higher linearity has been obtained in case of detection in water environment. Furthermore, a comparison of the sensor sensitivity in the two different environments in the case of toluene detection can be carried out in the concentration range $40-80 \mathrm{ppm}$ since in this range a linear behavior of the sensor calibration curve has been observed also for the case of detection in air. The results revealed a sensitivity in air higher (approx. $1.5 \cdot 10^{-4} \mathrm{ppm}^{-1}$ ) than that obtained in the case of water environment (approx. $\left.1.2 \cdot 10^{-4} \mathrm{ppm}^{-1}\right)$. The differences in the two characteristics can be attributed to the dependence of the reflectance upon the surrounding refractive index combined to different adsorption characteristics occurring in liquid environment, especially if the kinetic is diffusion limited. Taking into account the sensitivity of the sensor against toluene and considering the $\Delta R / R_{0 \min }\left(6 \cdot 10^{-4}\right)$ obtainable with the exploited instrumentation, a resolution of $5 \mathrm{ppm}$ has been estimated.

Finally, an analysis of the response $\left(t_{10-90}\right)$ and recovery $\left(t_{10-90}\right)$ times of the optical probes has been carried out. The results obtained for the detection in water are shown in Figure 19(b) and reveal characteristic times slightly dependent upon the analyte concentration, with the mean response time (approx. 20 minutes) higher than the mean recovery one (approx. 4 minutes). In Figure 20, a comparison between the mean characteristic times obtained with the SOF sensors coated by 12 monolayers of SWCNTs in the two 


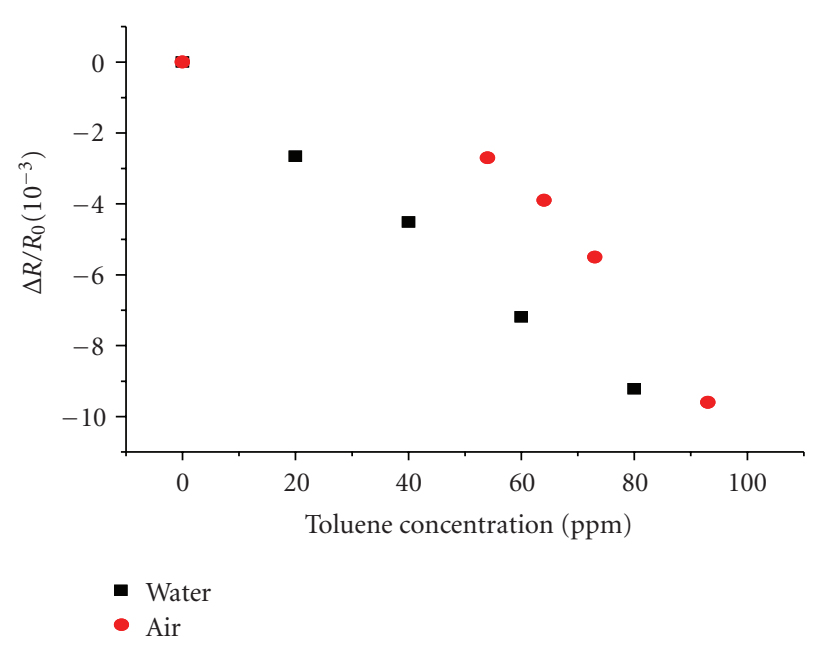

(a)

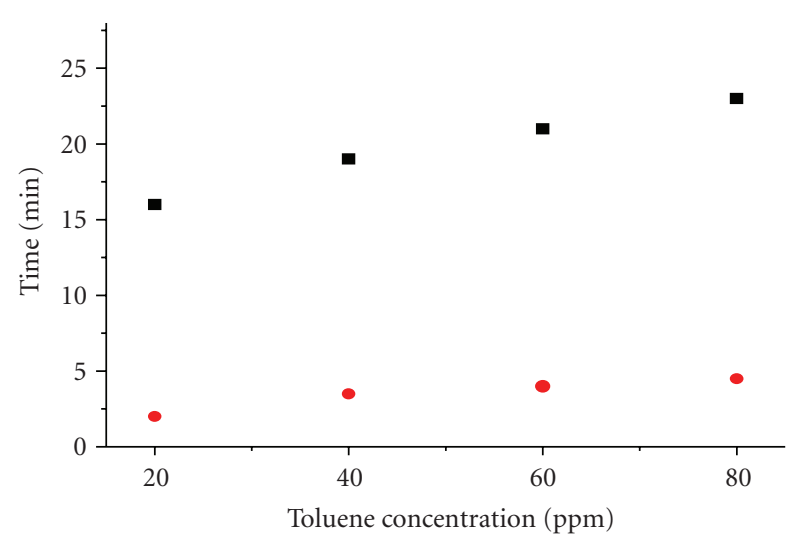

- Response times

- Recovery times

FIGURE 19: (a) Comparison between the calibration curves of the SOF sensor coated by 12 monolayers of SWCNTs against toluene obtained in air and aqueous environments; (b) response and recovery times of the SOF sensor, exposed to toluene with concentration in the range 20-80 ppm.

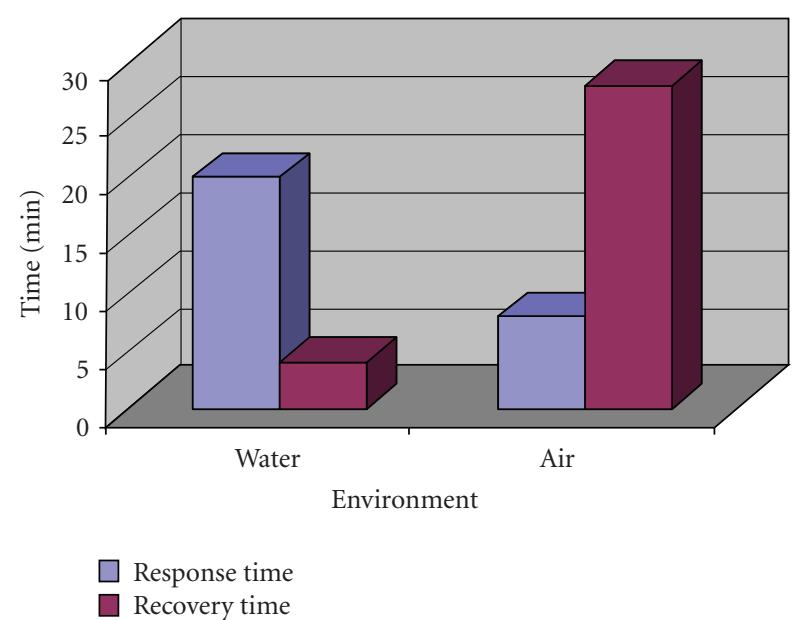

FIGURE 20: Comparison between the response and recovery times of the proposed SOF sensors in case of toluene detection in water and air.

different environments is reported. It can be seen that, also in this case, a difference has been observed with the response and recovery times obtained against toluene in air, which is approximately 8 minutes and approximately 28 minutes, respectively.

\subsection{Gaseous hydrogen detection at cryogenic temperatures}

The amazing characteristic of SWCNTs of being highly adsorbent materials has been successfully exploited also for the detection of gaseous hydrogen $\left(\mathrm{H}_{2}\right)$ at cryogenic temperatures. As a matter of fact, the experimental results provided in this section will reveal the excellent potentialities of the proposed SWCNTs-based optochemical sensors in this interesting environmental and industrial application.

Cryogenic $\mathrm{H}_{2}$ is often utilized as the primary fuel source by rocket engine because of the benefits deriving from hydrogen combustion properties. Unfortunately, gaseous hydrogen has a very low flammability limit and low ignition energy, so that it becomes a very dangerous and volatile substance to work with during the rocket engine development stage. Hydrogen leakage into an oxidizing environment can easily ignite a catastrophic explosion. For this reason, the development of accurate and reliable devices for the detection of hydrogen leakage at cryogenic temperatures is of crucial importance for human safety. In 1981, Lundström introduced the first $\mathrm{H}_{2}$ sensor based on thin films of Palladium (Pd) deposited on the top of the channel of an MOSFET device [73, 74]. Since then, this issue has been widely studied by the researchers and a number of hydrogen sensors have been proposed $[75,76]$. However, among the big amount of sensing devices, fiber optic detectors have strongly attracted the attention of the scientific community in this field, especially because of their high sensitivity and lack of sparking possibilities, guaranteed by the removal of all electrical power from the test site. To date, several configurations of fiber-optic hydrogen sensors have been proposed, based on different transducing principles and materials. [77, 78]. The most exploited material in this field is Pd since it exhibits strong thickness and refractive index changes on $\mathrm{H}_{2}$ molecule adsorption at room temperature. However, at cryogenic temperatures Pd looses its excellent sensing properties [79], thus the main effort in this field is devoted to the research of new sensitive materials that are able to provide high sensitivity and good desorption features 


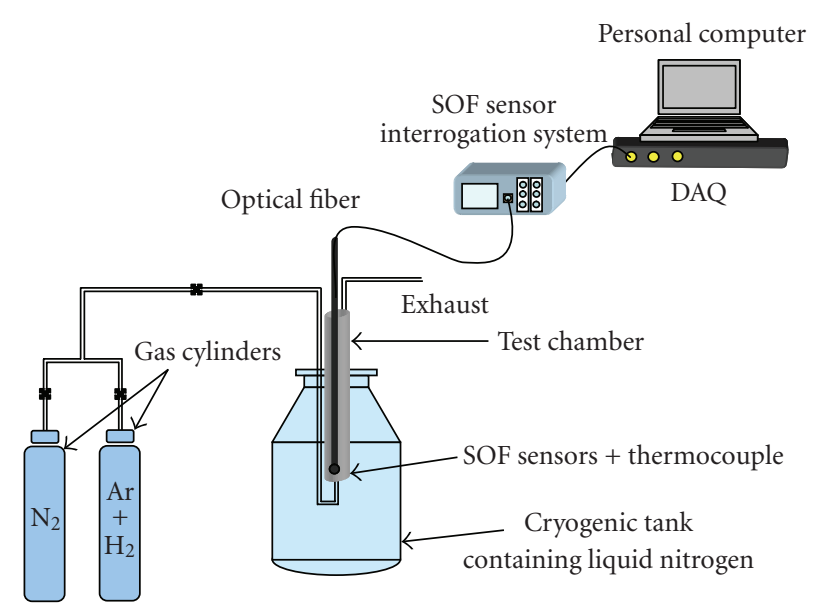

(a)

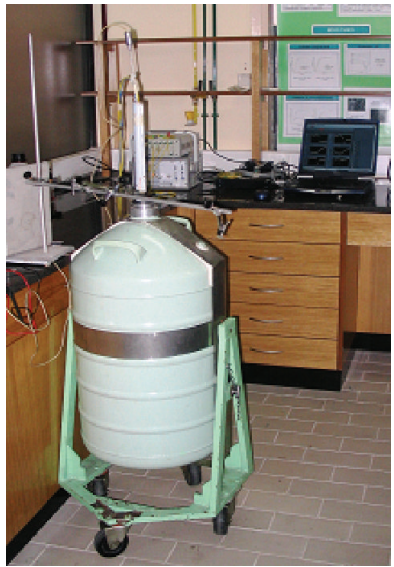

(b)

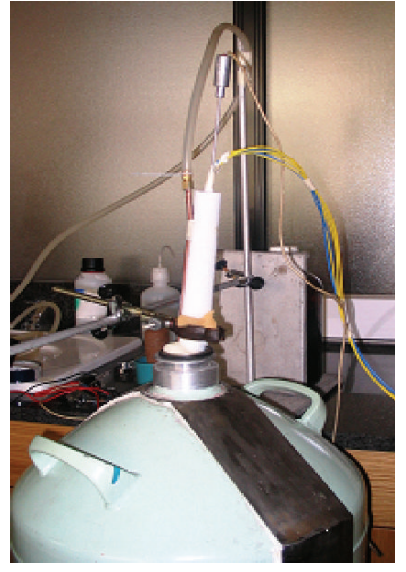

(c)

Figure 21: (a) Schematic view and (b), (c) images of the experimental setup exploited for the $\mathrm{H}_{2}$ detection at cryogenic temperatures.

as well as fast responses at very low temperatures. Recent reports have demonstrated a high reversible adsorption of molecular hydrogen in carbon nanotubes and graphitic nanofibers $[80,81]$. In light of this argument, SWCNTs (of both closed-end and opened-end types) have been exploited as sensitive nanostructured coatings of reflectometric SOF sensors for the detection of gaseous $\mathrm{H}_{2}$ at a temperature as low as $113 \mathrm{~K}$ [51]. In the following, the experimental results obtained by using three reflectometric sensors, coated respectively by two and six monolayers of closed-end SWCNTs (namely, 2_CeSWCNTs and 6_CeSWCNTs, resp.) and two monolayers of opened-end SWCNTs (2_OeSWCNTs), are reported.

In order to test their $\mathrm{H}_{2}$ sensing performance, the sensors were located in a cylindrical chamber, properly designed and realized in order to minimize the possible thermal variations. The chamber was, in turn, inserted within a tank containing cryogenic nitrogen (see Figure 21) with the aim of reaching the very low temperature of $113 \mathrm{~K}$.

The temperature has been monitored by a copper/ copper-nickel thermocouple located close to the optical sensor head. Nitrogen gas has been continuously injected in the cylindrical chamber in order to reduce the humidity content inside the test ambient before the strong temperature decreasing due to the insertion of the chamber in the cryogenic tank. To expose the SOF sensors to gaseous hydrogen with concentrations as low as $4 \%$ and $1 \%$, nitrogen has also been mixed with gaseous argon containing hydrogen.

The results obtained are shown in Figure 22, where the $\Delta R / R_{0}$ of the sensors (a) 2_CeSWCNTs, (b) 6_CeSWCNTs, and (c) 2_OeSWCNTs, exposed to decreasing concentration pulses of hydrogen at a temperature of $113 \mathrm{~K}$, have been reported. As evident, all the optical probes provided significant reflectance changes as a consequence of the gaseous $\mathrm{H}_{2}$ adsorption within the sensitive nanomaterials. In particular, good sensitivity and complete recovery of the nitrogen signal after both hydrogen concentration pulses have been observed for the two CeSWCNTs-based sensors, while good sensitivity but no recovery of the steady-state value has been observed for the OeSWCNTs-based one.

In addition, the bar plot shown in Figure 23, in which the amplitude of the relative reflectance changes $\left(\left|\Delta R / R_{0}\right|\right)$ obtained for the tested SWCNTs-based probes has been reported, clearly reveals that for the probe 2_CeSWCNTs, the $\Delta R / R_{0}$ obtained for $4 \%$ and $1 \%$ of $\mathrm{H}_{2}$ are almost similar (the ratio is approx. 1.1). In this case, a saturation of the fiber overlay sorption capability could explain the weak mismatch between the sensor responses.

Differently, for the case of the sensor 6_CeSWCNTs, a decrease of the $\mathrm{H}_{2}$ concentration of four times leads to a relative reflectance diminution of approximately three times. In this case, however, due to the increase of nanotubes monolayers, higher saturation threshold is expected.

Furthermore, a study of the response and recovery times $\left(t_{10-90}\right.$ and $t_{90-10}$, resp. $)$ has been carried out. The results obtained have been reported in Figure 24 and reveal that the probes 2_CeSWCNTs and 6_CeSWCNTs are characterized by the same response times, (approx. 4 minutes), but have slightly different recovery times (approx. 9 and 11 minutes, resp.). This evidences that the number of carbon nanotube monolayers influences the sensor recovery features. In addition, the probe 2_OeSWCNTs showed a response time (approx. 5 minutes) higher than that obtained with the CeSWCNTs-based sensors. This could be attributed to the fact that, while in the case of the CeSWCNTs, the $\mathrm{H}_{2}$ molecules adsorption takes place only on the surface of the single carbon tubes and in the interstitial sites between the tubes, in the case of the counterpart OeSWCNTs, the hydrogen molecule adsorption takes place also inside the carbon tubes, requiring, for this reason, more time for the establishment of the equilibrium condition with the external environment.

\subsection{Discussion}

Here, a brief discussion concerning some of the typical drawbacks arising from the use of carbon nanotubes as 


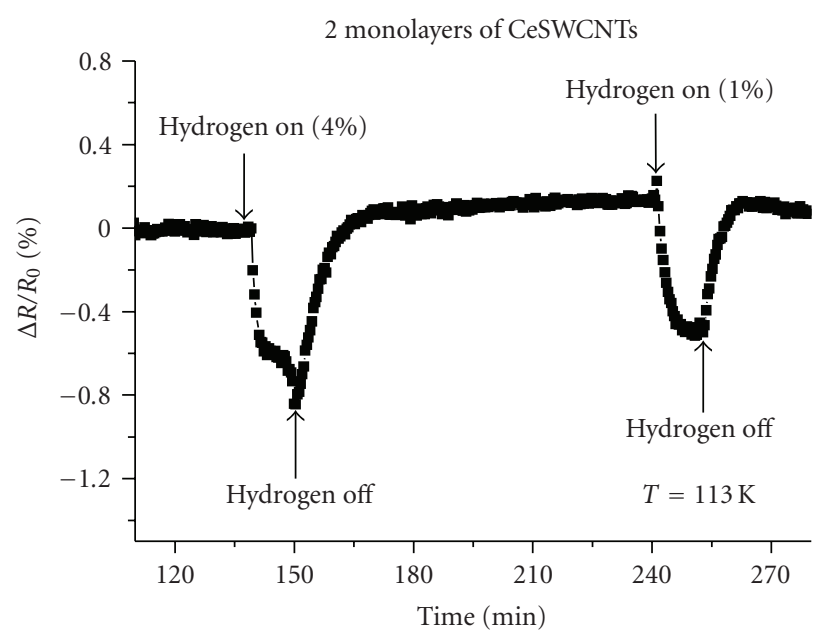

(a)

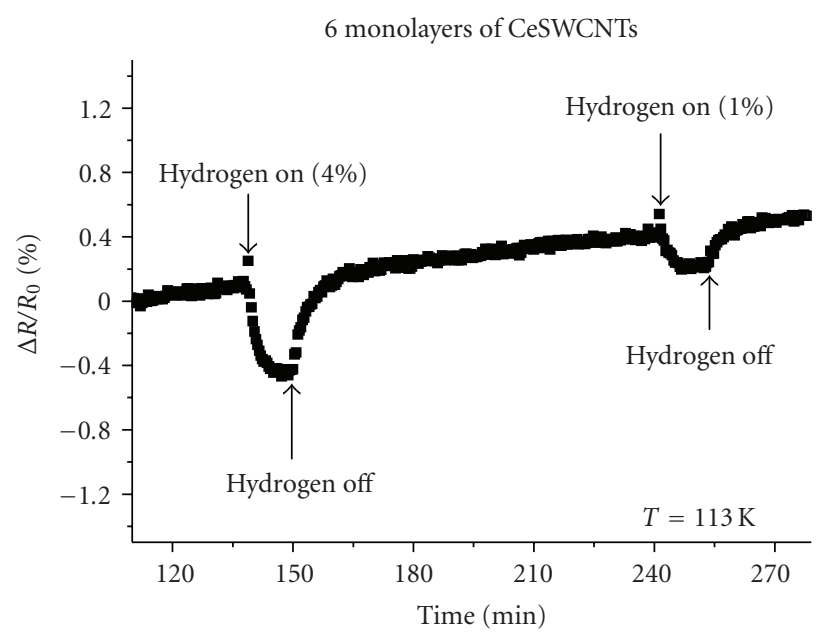

(b)

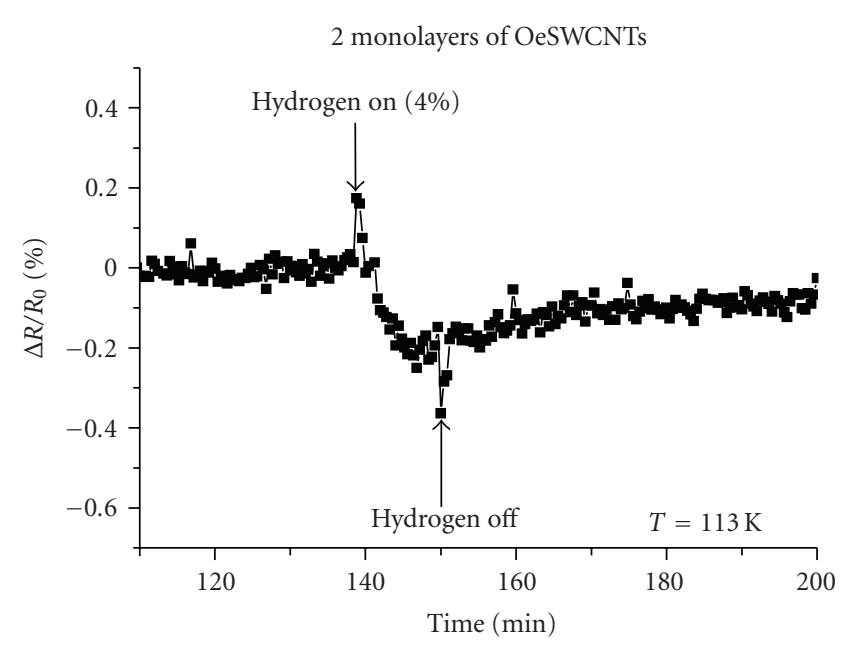

(c)

FIgURE 22: Transient responses of the three fiber optic probes (a) 2_CeWCNTs, (b) 6_CeWCNTs, and (c) 2_OeWCNT to decreasing concentration pulses of gaseous hydrogen $(<5 \%)$, at $113 \mathrm{~K}$.

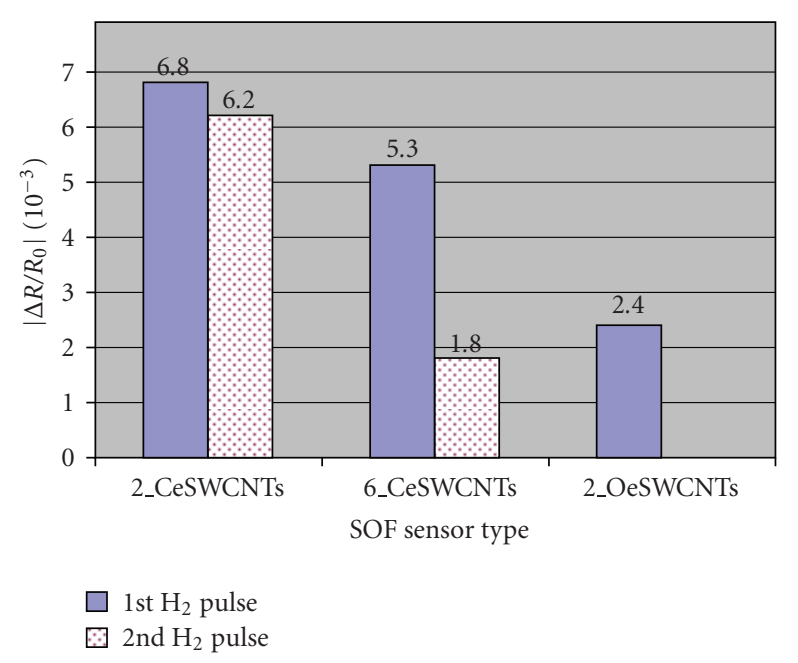

FIGURE 23: $\Delta R / R_{0}$ occurred to the three tested SOF sensors, on $\mathrm{H}_{2}$ exposure.

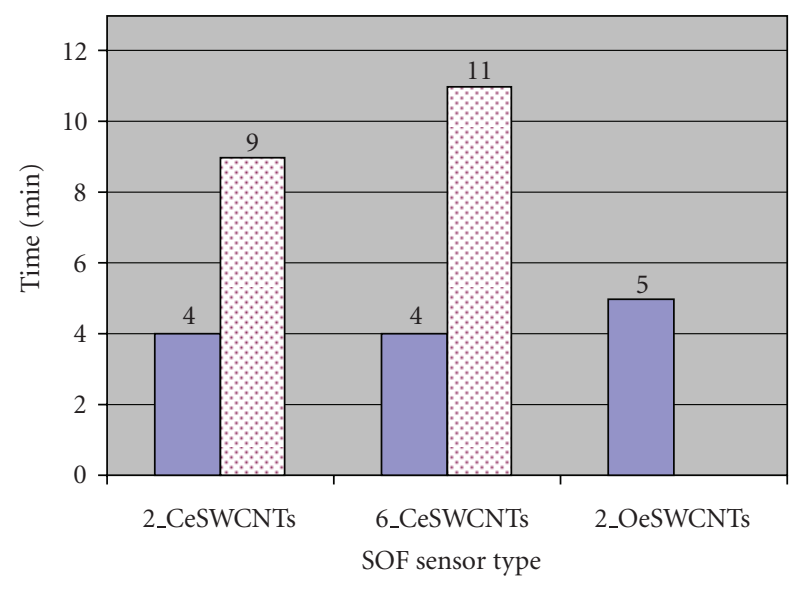

Response time

Recovery time

FIGURE 24: Mean response and recovery times for the three tested SOF sensors.

sensitive elements of reflectometric optical fiber sensors is carried out, which evidences some currently unsolved matters, but, at the same time, opens the way to further investigations and research activities.

First of all, the attitude of SWCNTs to randomly distribute in tangled networks of nanotubular chains and densely aggregate mats causes a not very high repeatability of the distribution of carbon nanotubes upon the sensor surface as well as their optical properties. These drawbacks could be overcome by means of an alignment of the carbon nanotubes onto the sensor substrate, which should provide a better and almost predictable SWCNT distribution upon them.

The adhesion of the carbon nanotubes upon the fiber substrate also requires further investigations and improvements, especially when the SWCNTs-based sensors are exploited for in water chemical detection applications, for 
which particularly hard operating conditions could even promote a detachment of the sensitive overlay from the optical fiber tip. A first attempt to improve the adhesion of SWCNT overlays to the distal end of the optical fibers is reported in the next section and relies on their embedding in CdA host-matrix in order to obtain nanocomposites with tailored amount of nanotube filler contents.

Finally, another issue to address is related to the poor selectivity of the proposed SWCNTs-based sensors towards a given chemical specie. This means that in case of a multicomponent gas mixture with interfering analytes, the information provided by the sensors could be ambiguous and no straightforward information about the test environment could be achieved. This is a very common aspect in chemical sensing applications as demonstrated by the strong effort which is currently devoted by the researchers on how to improve the sensor discrimination abilities among different analytes. Basically, there are two possible approaches in the attempt to increase the chemical sensor selectivity, and both have to be followed in synergy. The first one is a direct approach which relies on the sensitive layer functionalization in order to have a higher affinity of the material towards specific chemical species [82]. The second one is based on the use of a hybrid system composed of multiple transducers coated by the same material (or, in the most general case, by different materials) in the form of an array. Here, sensors with a poor selective response, when considered collectively, provide unique patterns typical for each analyte. The generated response patterns are interpreted by patternrecognition algorithm for the selective detection [83]. This approach has already been investigated and exploited by the authors [50], and is currently under investigation.

\section{SWCNTS-BASED NANOCOMPOSITES AS ADVANCED NANOCOATINGS}

As already discussed in Section 4.4, the adhesion of the carbon nanotubes to the fiber substrate and the poor repeatability of the deposition process (especially concerning the distribution of the tubes upon the fiber tip as well as the optical properties of the fabricated layer) represent two important issues to be improved for an extensive use of the proposed SWCNTs-based optochemical sensors for practical applications and for their effective transfer to the market. The alignment of carbon nanotubes upon the sensors substrate and the embedding of controlled quantity of them inside a host-matrix of a foreign material for the synthesis of nanocomposites with tailored amount of nanotube filler contents have been identified as possible ways to overcome these drawbacks. In fact, the CdA was used in the past as buffer-linker material to promote the adhesion of SWCNT thin films to the fiber end-face. Here the attention has been focused on the latter solution. In particular, the sensing performances of fiber optic chemosensors based on nanocomposite overlays of SWCNTs embedded in a CdA matrix have been investigated against several chemical pollutants both in air and water environments, at room temperature.

\subsection{Fabrication and characterization of CdA/SWCNTs-based fiber optic chemosensors}

The Langmuir-Blodgett deposition process has been exploited again as a way to transfer thin films of SWCNTsbased nanocomposites upon the distal end of properly prepared single-mode optical fibers. The CdA has been chosen as host-matrix material to incorporate the SWCNTs in the nanocomposite due to its peculiar amphiphilic molecular structure suitable for LB deposition process and because of the know-how already experienced by the authors in the integration of such material and the optical fiber transducers $[49,50]$. Two separate solutions of arachidic acid in chloroform and SWCNTs in chloroform have been mixed in order to prepare a final solution of chloroform with arachidic acid $(0.25 \mathrm{mg} / \mathrm{mL})$ and SWCNTs $(0.19 \mathrm{mg} / \mathrm{mL})$. The concentrations and the volumes of the initial solutions were chosen to obtain a weight percentage of the filler component (SWCNTs) with respect to the matrixcomponent (CdA) of approximately $75 \mathrm{wt} . \%$. However, different concentrations of arachidic acid and SWCNTs in the final solution could also be exploited for the preparation of composites with different weight percentages. The mixed solution was then accurately dispersed and stirred in an ultrasonic bath for 1 hour. Subsequentially, $160 \mu \mathrm{L}$ of the mixed solution were spread onto a subphase constituted by acetate buffer with $\mathrm{CdCl}_{2} 10^{-4} \mathrm{M}$. The $\mathrm{pH}$ and the temperature of the subphase were kept constant at 6.0 and $20^{\circ} \mathrm{C}$, respectively. The monolayer of the nanocomposite was compressed with a barrier rate of $15 \mathrm{~mm} / \mathrm{min}$ up to a surface pressure of $27 \mathrm{mN} / \mathrm{m}$. The single composite monolayer was deposited upon the fiber surface with a dipping rate of $14 \mathrm{~mm} / \mathrm{min}$. The optical fibers used for the deposition have been previously accurately polished from the acrylic protection and cleaved with a precision cleaver. Then, they have been washed in chloroform to remove any residual coating. Repeated dipping of the fiber substrates through the condensed Langmuir layer has been performed, resulting in the deposition of multilayered CdA/SWCNT films one monolayer at a time.

Figure 25(a) shows the typical SEM photogram of a multilayer constituted by twenty monolayers of CdA/SWCNT nanocomposite with a filler weight percentage of $75 \mathrm{wt} . \%$ (deposited upon a glass substrate). The typical bundle disposition of the carbon nanotubes together with their good cadmium arachidate-assisted adhesion to the surface can be easily observed. A detailed morphological and structural characterization of the carbon nanotubesbased composites deposited by the LB method has been carried out by Penza et al. [84]. Instead, here, the results of Raman spectroscopy analysis are reported, conducted with the aim of characterizing the fabricated nanolayers of CdA/SWCNTs (already deposited upon the optical fiber tip) and comparing them with the standard SWCNTs one. To achieve this purpose, a Raman microscope functioning in backscattering configuration employing an $\mathrm{HeNe}$ laser $(633 \mathrm{~nm})$ and 50x and 100x objective lenses was used. The results are shown in Figure 25(b), where the typical Raman 


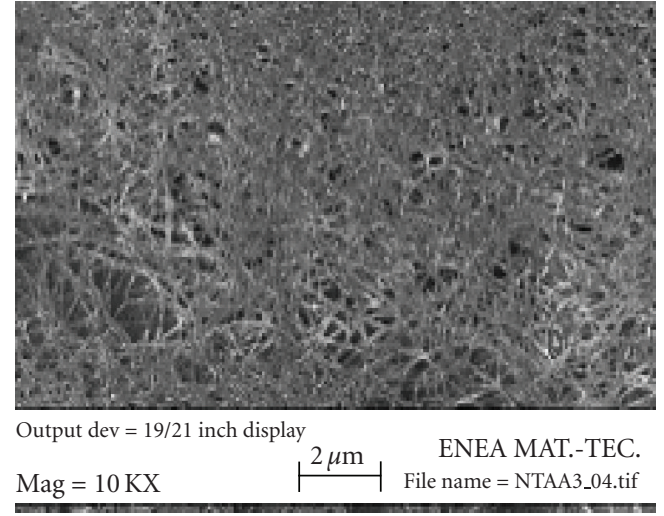

(a)

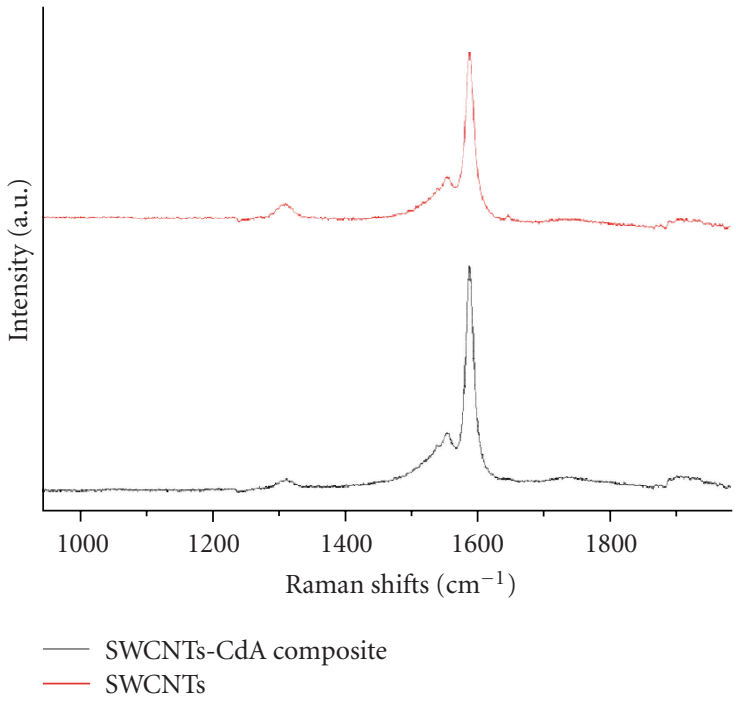

(b)

FIGURE 25: (a) SEM image of a CDA/SWCNT overlay with a filler content percentage of 75 wt.\%, deposited upon a glass substrate; (b) typical Raman spectra of SWCNT and CdA/SWCNT LB overlays directly deposited upon the optical fiber end-face.

spectra of SWCNTs and carbon nanotubes-based composite layers, already deposited upon the fiber end-face, have been reported.

No significant differences between the two recorded Raman spectra can be observed, thus evidencing that no degradation of the CNT occurred due to their inclusion within the CdA matrix.

\subsection{Room temperature toluene and xylene vapor detection}

The room temperature sensing performance of the fabricated probes have been investigated both in air and water environments and have been compared with the ones obtained with the same transducers coated by unbuffered SWCNT overlays. Here the attention is focused on the toluene and xylene vapors detection by using a sample coated by 20 monolayers of nanotubes-based composite with an SWCNT filler weight percentage of $75 \mathrm{wt} . \%$. The results obtained from the experimental measurements are shown in Figure 26. Here the relative reflectance changes occurred as a consequence of the exposure to (a) toluene and (b) xylene vapors have been reported. The analyte adsorption within the SWCNTs-based nanocomposite overlay promoted significant changes of the optical length of the FP sensing cavity (defined as the product between its thickness and refractive index), resulting in a decrease of the fiber-film reflectance. The results also reveal the capability of the optoelectronic transducer to detect very low concentrations of the tested pollutants at ppm levels, as well as its quite good attitude to recover the initial baseline signal upon the complete analyte molecules desorption.

Furthermore, an almost linear behavior in the sensor calibration curves (reported in Figure 27) has been found towards the two organic vapors in the investigated ranges (0-83 ppm and 0-44 ppm for toluene and xylene, resp.), and a higher sensitivity against xylene vapors $\left(1.2 \cdot 10^{-3} \mathrm{ppm}^{-1}\right)$ was obtained with respect to toluene ones $\left(5 \cdot 10^{-4} \mathrm{ppm}^{-1}\right)$.

Also, an analysis of the mean response $\left(t_{10-90}\right)$ and recovery $\left(t_{90-10}\right)$ times (see Figure 28 ) revealed that the optochemical probe coated by twenty monolayers of CdA/SWCNT nanocomposite is characterized by a faster dynamic in case of toluene exposure ( 32 and 39 minutes, resp.) than in the xylene one (36 and 41 minutes, resp.). It is noteworthy that the relatively high response times can be attributed to the high number of composite monolayers (and thus to the overlay thickness used for the sensor fabrication). Also, the content of SWCNTs can influence the diffusion times as well as the sensor sensitivity. These aspects, however, are still under investigation.

The sensing performance of the fabricated probe can be compared with the results obtained with the probe coated by four monolayers of SWCNTs directly deposited upon the fiber end (SOF-4), which demonstrated toluene and xylene sensitivities of approximately $4.7 \cdot 10^{-4} \mathrm{ppm}^{-1}$ and $1.1 \cdot 10^{-3} \mathrm{ppm}^{-1}$ and mean response times of 9 and 11 minutes, respectively (see Section 4.1) [6]. The results obtained reveal that slightly higher sensitivities have been obtained by using CdA/SWCNT composites, combined with longer mean response times, probably due to the different number of monolayers and overlay composition in the two cases.

\subsection{Chemical trace detection in aqueous environments}

Once their excellent VOC adsorption capabilities in air at room temperature have been verified, the sensing characteristics of the CdA/SWCNT nanocomposite have been investigated also for the aromatic hydrocarbon detection in aqueous environment. To this aim, the same sensor used 


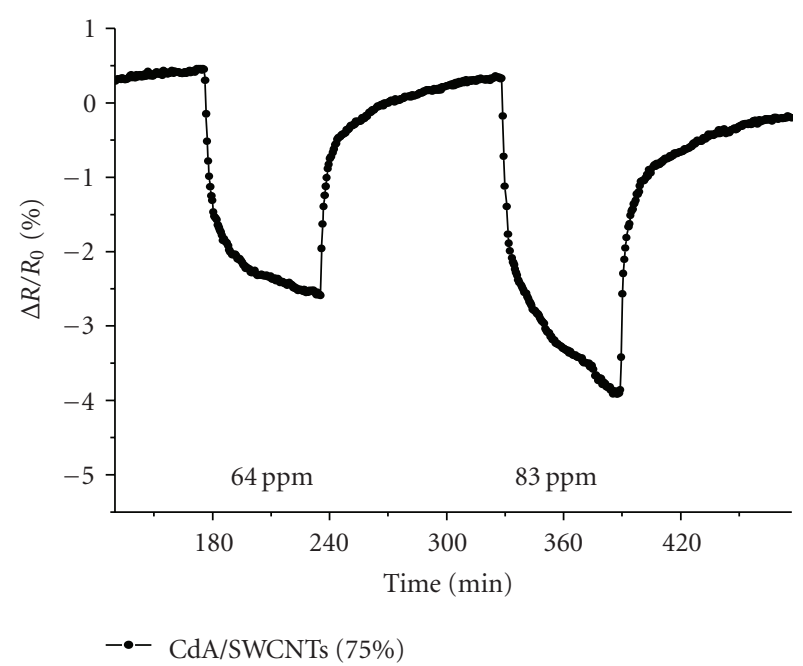

(a)

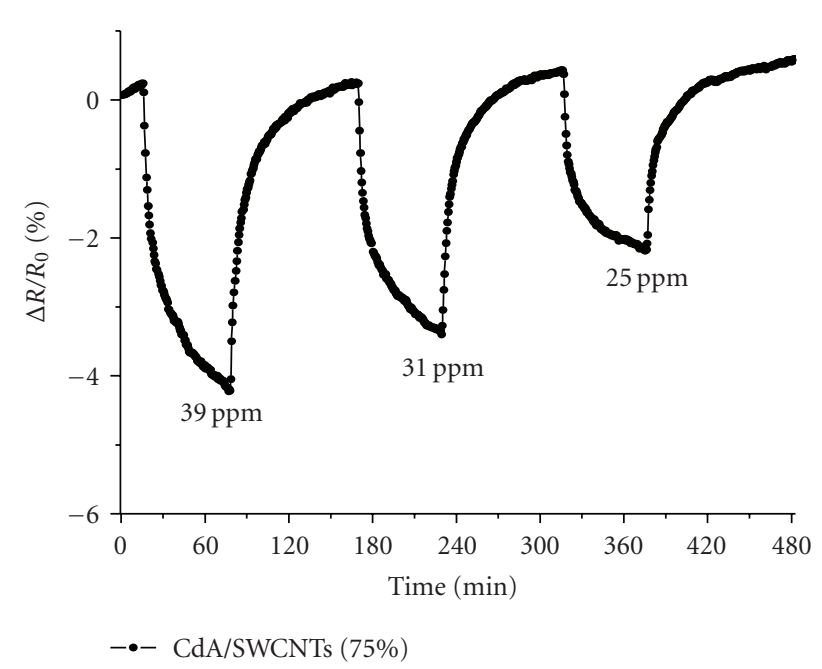

(b)

FIGURE 26: Time response of the optochemical sensor coated by 20 monolayers of CdA/SWCNT nanocomposite (75 wt.\%), exposed to different concentrations of (a) toluene and (b) xylene vapors, at room temperature.

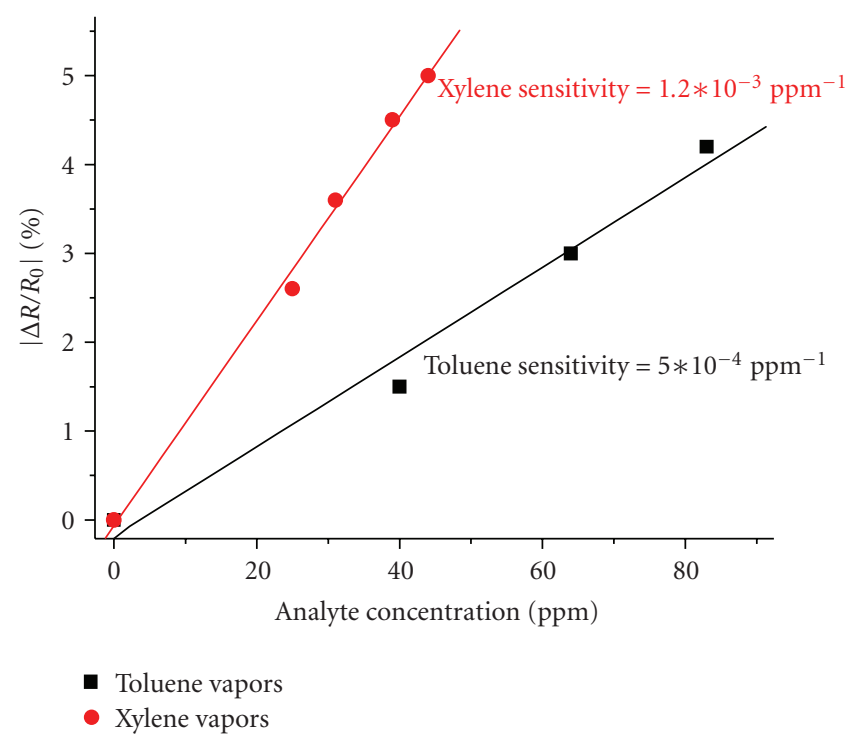

FIgURE 27: Comparison between the sensor characteristic curves obtained for toluene and xylene vapors.

in air environment was tested for the detection of low concentrations of toluene and xylene in water by using the experimental setup already reported and explained in detail in Section 4.2. Figure 29 reports the transient responses of the fiber optic probe as a consequence of several toluene injections with concentrations ranging from 20 to $100 \mathrm{ppm}$ $(\mu \mathrm{L} / \mathrm{L})$. Like in the air case, a significant reflectance decrease occurred on analyte adsorption within the sensing nanolayer. In addition, a good repeatability has been observed in the sensor response when exposed to two successive $100 \mathrm{ppm}$ xylene injections.

Furthermore, Figure 30 demonstrates that also in the case of detection in aqueous ambient a linear dependence

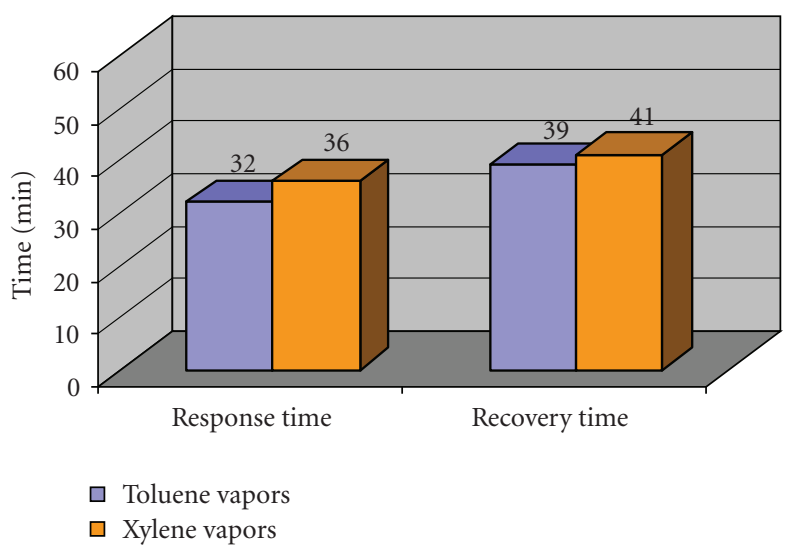

FIGURE 28: Mean response and recovery times obtained exposing the optochemical sensor coated by 20 monolayers of CdA/SWCNT nanocomposite $(75 \mathrm{wt} . \%)$ to toluene and xylene vapors, at room temperature.

exists between the fiber-film reflectance change and the concentration of the two organic analytes, with a higher affinity towards xylene (the sensor sensitivity is $1.0 \cdot 10^{-3} \mathrm{ppm}^{-1}$ ) than toluene $\left(4 \cdot 10^{-4} \mathrm{ppm}^{-1}\right)$. These sensitivities are slightly lower than those obtained for the chemical detection in air (see the previous section).

However, strong differences have been observed between the dynamics of the sensor responses in the two environments, with the mean response and recovery times against toluene (11 and 7 minutes, resp.) and xylene (14 and 7 minutes) in water (see Figure 31) considerably lower than the ones evidenced by the CdA/SWCNTs-based sensor in air (this effect could be due to the difference of the two test chambers and of the diffusion kinetic in the two environments). Finally, in aqueous ambient the proposed 


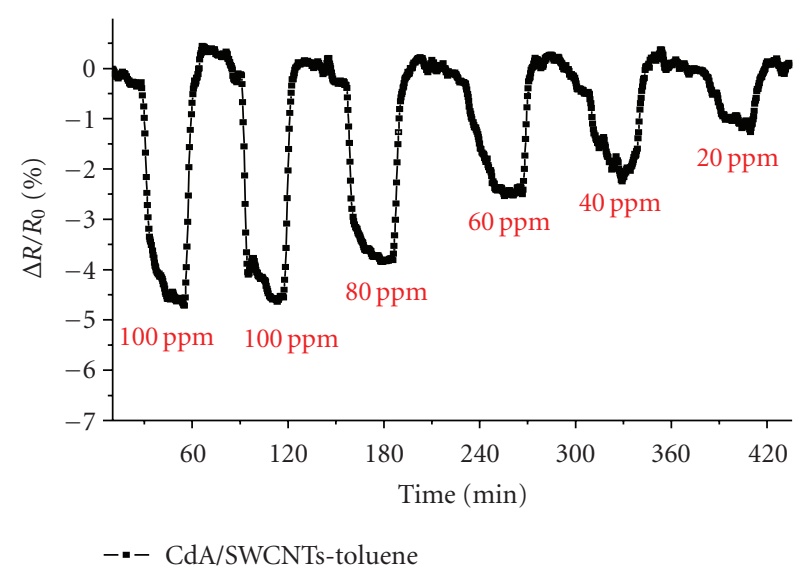

FIgURE 29: Time responses of the optochemical sensor coated by 20 monolayers of CdA/SWCNT nanocomposite (75 wt.\%), exposed to different concentrations of toluene in water at room temperature.

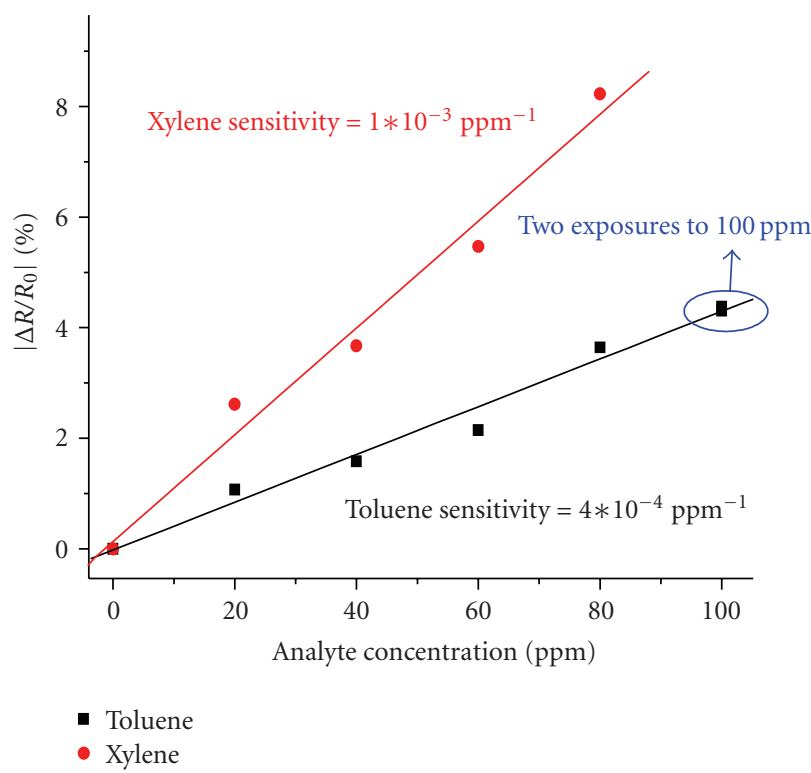

FIgURE 30: Comparison between the sensor characteristic curves obtained in correspondence of toluene and xylene injections in water environment.

sensor exhibited enhanced performances with respect to unbuffered SWCNT films especially in terms of sensor sensitivity, for which enhancement of four times has been observed [52].

\section{HOLLOW-CORE FIBER OPTIC SENSORS: TOWARDS ADVANCED SENSING CONFIGURATIONS}

In this last section, a novel sensing scheme is presented, based on the integration of SWCNTs with special fibers, the hollow-core optical fibers (HOFs). HOFs are composed of bidimensional arrays of air holes of micrometric dimensions running along the fiber length in which the light is confined within the air core by means of the photonic bandgap (PBG) constituted by the microstructured cladding. This special

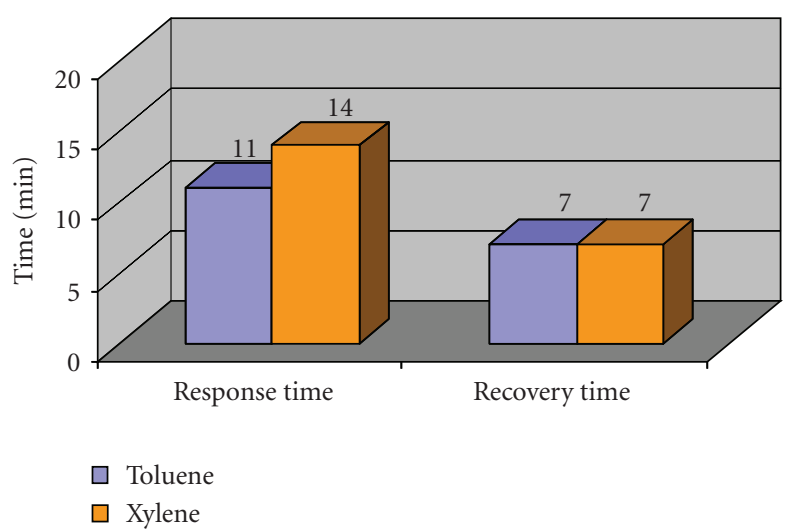

FIGURE 31: Mean response and recovery times obtained exposing the optochemical sensor coated by 20 monolayers of CdA/SWCNT nanocomposite $(75 \mathrm{wt} . \%)$ to toluene and xylene in water, at room temperature.

feature of the HOFs allows one to develop new functionalized devices by filling the fiber holes with proper materials. As a matter of fact, the integration between HOFs and specific materials enables the final device to own advanced properties in terms of tunability [85-87] and sensitivity [53]. In particular, it can lead to the development of novel optoelectronic chemosensors that exploit the changes of the HOF guiding properties due to the presence of the sensitive material within the holey structure [53]. The experimental data here reported will demonstrate the success of the SWCNT partial filling within the HOF holes and the capability of the novel sensing devices thus formed to detect VOC traces.

\subsection{HOF sensor fabrication and characterization}

With reference to Figure 32, the HOFs-based sensing probes are composed of a piece of HOF, spliced at one end with an SOF and covered and partially filled with SWCNTs at the other termination. The SWCNT deposition, performed before the HOF-SOF splicing, has been carried out at atmospheric pressure by means of the molecular engineered LB technique (see Section 3.3).

Similarly to any reflectometric configuration, also in this case the sensing principle relies basically on the dependence of the sensing probe reflectance on the optical properties of the sensitive materials. Nevertheless, in this case, the key factor in the determination of the sensing performance is the dependence of the sensing probe reflectance on the guiding properties of the portion of HOF filled with SWCNTs.

The injection of the LB suspension inside the HOF holes and thus the adhesion of carbon nanotubes onto the airhole sides were guaranteed by the dipping of a piece of HOF, having one termination not connected (atmospheric pressure), through the CNT suspension, perpendicularly to it for a depth of approximately $1 \mathrm{~mm}$ [88]. For the HOFSOF splicing, the open end of each HOF piece has been spliced to the input end of an SOF by using an electrical arc discharge system (Fujikura FSM-50S). The fiber ends, 


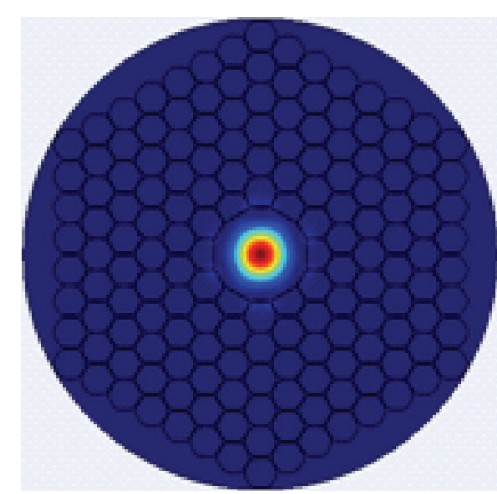

(a)

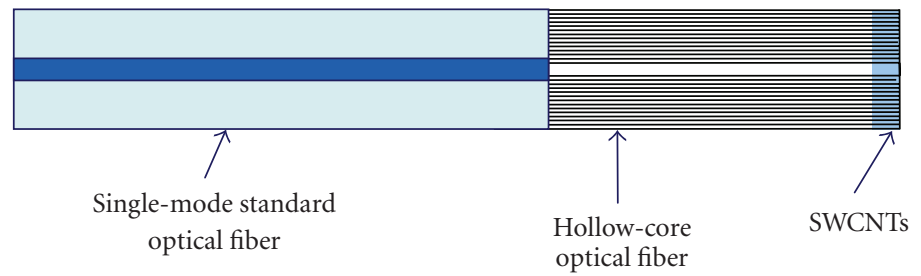

(b)

Figure 32: (a) Schematic view of the transverse section of an HOF and its fundamental mode field distribution; (b) schematic view of HOF-based sensor probe.

both cleaved, have been aligned and pressed against each other using the splicer precision motors. A proper splicing procedure, involving a series of ten arcs with short duration (100 $\mathrm{ms})$ and high power ( $80 \mathrm{bit})$ [53], has been developed to avoid the collapse of the air holes. The process was previously tested and the losses were estimated to be approximately $1 \mathrm{~dB}$ by comparing the power transmitted in butt coupling configuration with that after the optical fiber fusion. In addition, the bonding achieved between the two fibers, weak with respect to the bending, has been enforced by using a heating protection sleeve. Several sensors have been realized with this technique by varying the fabrication features such as the HOF length and the number of SWCNT monolayers. However, here an extensive analysis of two samples is reported, the former one (namely SENSOR 1) constituted of a $13.3 \mathrm{~cm}$ long HOF coated by ten monolayer of SWCNTs; the latter (namely SENSOR 2) constituted of a $4 \mathrm{~cm}$ long HOF coated by twenty SWCNTs monolayers.

In order to investigate the typical morphological characteristics of the fabricated HOF sensors, several samples have been analyzed by means of SEM imaging. The retrieved images showed quite uniform CNTs-based overlays covering the fiber holes. The typical attitude of SWCNTs to randomly aggregate mats was revealed also in this case. As an example, Figure 33 reports the SEM image of an HOF before and after the deposition of sixteen monolayers of SWCNTs.

To better evidence the SWCNT distribution, only a detail of the HOF cladding has been shown, revealing an ultrathin weblike overlay covering the holes of the microstructured fiber. This also demonstrates the success of the carbon nanotubes integration with the HOFs.

A reflectance characterization of the realized probes have also been carried out within the HOF bandwidth by using a tunable laser source and an optical spectrum analyzer in synchronous mode allowing $1 \mathrm{pm}$ wavelength resolution in the spectral range $1520-1620 \mathrm{~nm}$. The reflectance spectra obtained for the two probes under investigation (reported in Figure 34) exhibit several interference fringes and also their envelope offers a quite periodic behavior. In particular, with regard to SENSOR 1, two different harmonic contents (fast and slow) can be clearly distinguished, suggesting the presence of a double cavity interferometer. From the wavelength separation of the fringes, the optical path length weighted by the medium effective refractive index of each interferometer can be retrieved according to the following equation [89]:

$$
2 \cdot n_{\mathrm{eq}} \cdot d=\frac{\lambda_{1} \cdot \lambda_{2}}{\left(\lambda_{2}-\lambda_{1}\right)},
$$

where $d$ is the length of the interferometer, $n_{\mathrm{eq}}$ is the medium effective refractive index of the propagating mode, $\lambda_{1}$ and $\lambda_{2}$ are the wavelengths corresponding to two adjacent maxima.

By applying (7) at the reflectance spectrum of SENSOR 1 , optical path lengths weighted by the medium effective refractive index of approximately $26.6 \mathrm{~cm}$ and $40 \mu \mathrm{m}$ have been estimated. The former one coincides approximately with the double length of the HOF piece (considering the effective refractive index of the HOF not filled by SWCNTs equal to 1). The latter one represents the extension of the region, on micrometric scale, constituted by the deposited SWCNTs, which are either within the HOF holes or outside of the HOF end face. The two cavities, hence, can be attributed to the piece of HOF and to the SWCNT region. In turn, from the analysis of the reflectance of SENSOR 2 , although the fast oscillating component is again clearly observable, the envelope of the reflectance seems to be periodic mostly in the wavelength range $1545-1575 \mathrm{~nm}$ while it is less recognizable a periodic behavior for the envelope in the remaining spectral range. Nevertheless, optical path lengths weighted by the medium effective refractive index of approximately $8 \mathrm{~cm}$ and $460 \mu \mathrm{m}$ have been estimated. The fast oscillating behavior is still attributed to the piece of HOF, which is approximately $4 \mathrm{~cm}$ long, whereas the SWCNTs seem to cover a region of hundreds of microns consistently with the larger amount of SWCNTs deposited in this latter case (twenty monolayers). Also, on the basis of previous works dealing with the SWCNT deposition onto the end-face of standard fibers, it results that ten and twenty monolayers of SWCNTs are not able to produce an external cavity able to produce interference fringes in the 


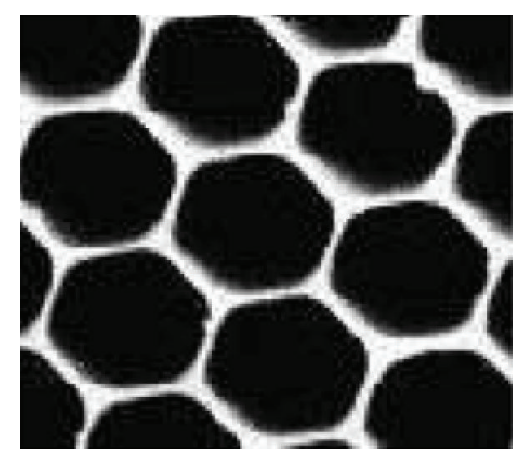

(a)

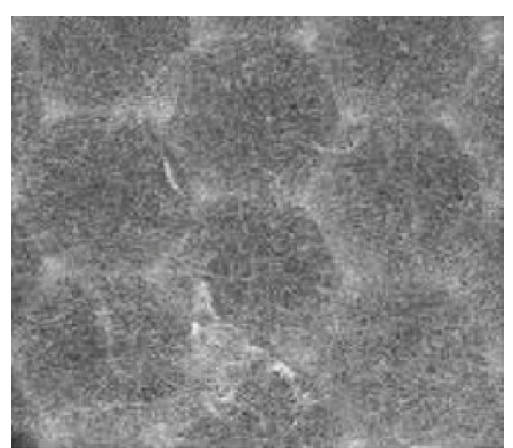

(b)

Figure 33: SEM image of an HOF (a) before and (b) after the deposition of sixteen monolayers of SWCNTs.

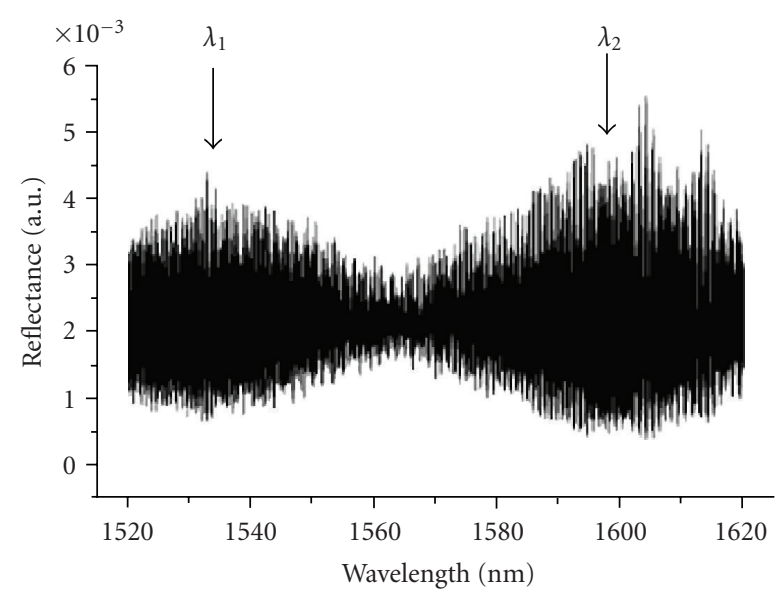

(a)

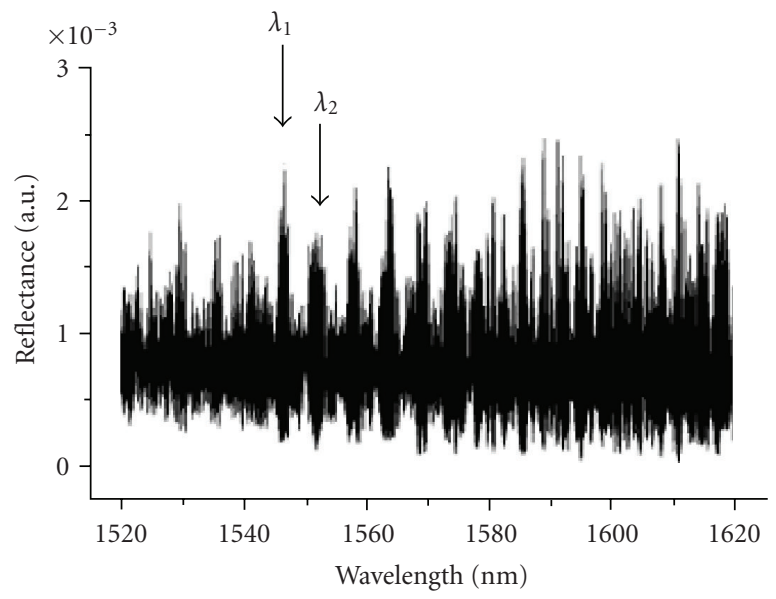

(b)

FIGURE 34: Reflectance of (a) SENSOR 1 and (b) SENSOR 2. The arrows indicate the wavelengths corresponding to two adjacent envelope relative maxima.

investigated wavelength range. Hence, the results obtained from the spectral characterization of both sensors reveal that the carbon nanotubes penetrated at least into the $\mathrm{HOF}$ central hole and their distribution along the HOF axis, which however cannot be assumed spatially uniform, extends for tens and hundred of microns, respectively. It is noteworthy that (7) is rigorously valid under the hypothesis of transparent media which in turn is not truly verified for carbon nanotubes-based materials. In addition, the estimation of the equivalent cavity length by means of the interference fringes together with the trivial measurement of the length of the HOF piece before the deposition is not able to discriminate the fraction of nanotubes penetrated inside the holes from the fraction of nanotubes which composes an external overlay at HOF termination [53]. Nevertheless, an insight of the double interferometer behavior of the sensing probe is obtained and an approximated estimation of the extension of the SWCNTs region can be retrieved.

The far field emerging from the fabricated sensors has also been collected by means of an infrared vidicon camera
(Hamamatsu C2741-03) while a narrowband laser source at $1550 \mathrm{~nm}$ lighted the sensing probes. The end face of the samples has been positioned in the nearby of the receiving lens. In Figure 35, the far field transmittances of SENSOR 1 and SENSOR 2 have been reported, respectively. In the case of SENSOR 1, it presents the typical quasi-Gaussian shape of the field associated to the HOF fundamental mode, which has been reported for comparison in Figure 35(c). At the bottom of the Gaussian waveform, little peaks of circular shape are observable, corresponding to the cladding modes also observed in the far field plot of Figure 35(c). The presence of the SWCNTs hence is not able to strongly modify the field distribution of the propagating mode while it confers the double interferometric behavior evidenced before. In addition, a slightly higher light content can be observed in correspondence of the HOF cladding in the coated case. This effect demonstrates a slightly worse confinement of the filled HOF consistent with refractive index contrast reduction induced by a partial filling of the cladding holes. 


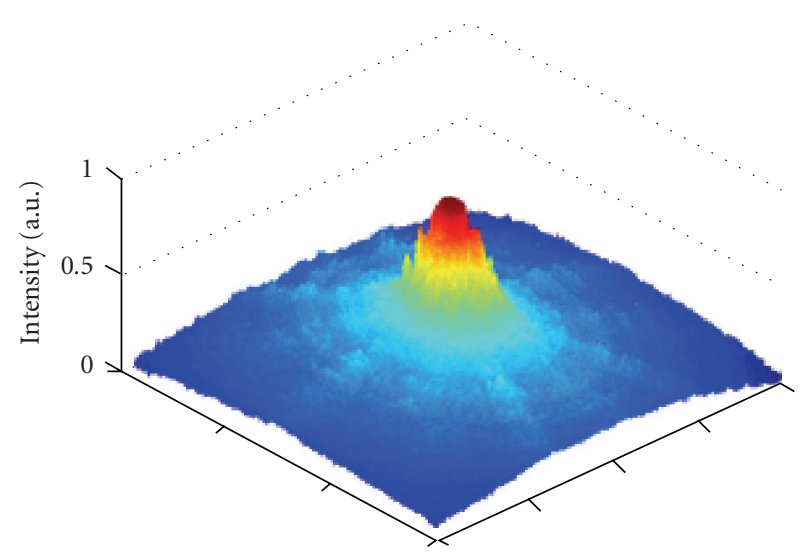

(a)

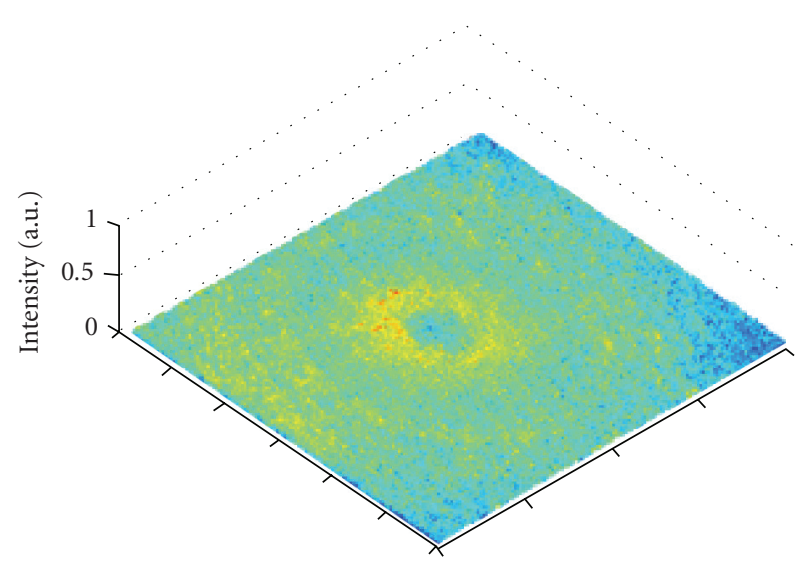

(b)

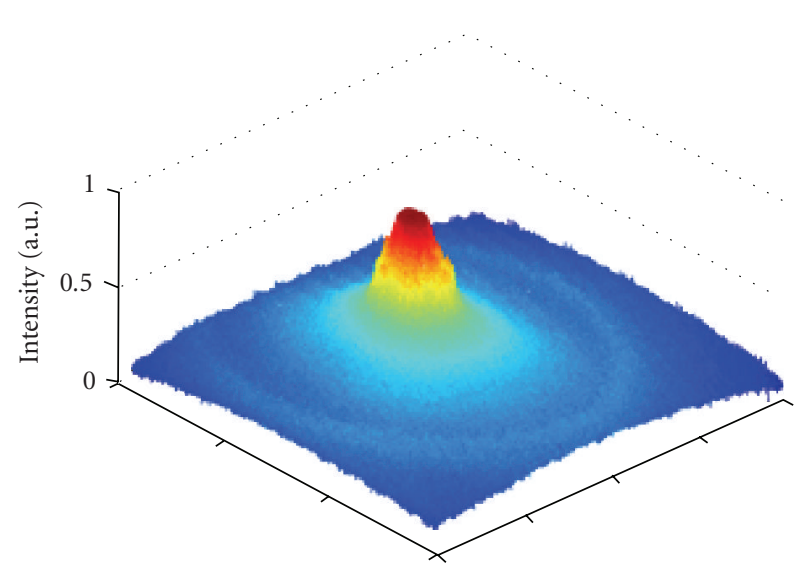

(c)

FIgURE 35: Distribution of the far field transmittances of (a) SENSOR 1, (b) SENSOR 2, and (c) a bare HOF.

With regards to SENSOR 2, instead, a strong modification of the field distribution occurs and the amount of power transmitted is strongly reduced. The emerging field presents a circular crown shape and the core mode is not more visible. In other words, the larger amount of SWCNTs penetrated within the holes are able to suppress the fundamental core mode, thus leading to a strong diminution of the confinement power. This circumstance is attributed to the modification of the PBG occurring as a consequence of the cladding and core hole filling which make the functionalized $\mathrm{HOF}$ not more able to guide meaningfully the light. The PBG modification affects particularly SENSOR 2 which was fabricated with a higher number of monolayers with respect to SENSOR 1. As a consequence, an overall deeper infiltration of the carbon nanotubes and also their higher density within the HOF holes occurred. Nevertheless, on the basis of the aforementioned reflectance characterization, it results that the light going through the sensing probe 2 experiences a double interferometer as far as the reflectance spectrum shows an envelope almost periodic. In light of the absence of the fundamental mode in the transmitted far field and in combination with the reflectance characterization, it can be deduced that the propagating mode going through the HOF filled with SWCNTs is not just reflected at the HOF-SWCNTs interface but it experiences along the SWCNT region a distributed mirror with losses before being suppressed. Consequently, the extension of the SWCNT region within the HOF could be underestimated. The penetration depth of the SWCNTs within the holes and their spatial distribution are currently under investigation as well as the effects of the deposition parameters on the resulting functionalized HOF and it will be the object of further work. In summary, the far field characterizations in combination with the spectral characterizations reveal the success of the SWCNT deposition onto the distal end of the HOF [53] and demonstrate the partial filling of the nanotubes within the microstructured holey-core optical fibers. Also, the obtained results show how by a proper control of the filling procedure, especially in terms of spatial distribution, it is possible to modify the PBG and thus the propagation properties of HOFs for specific applications.

\subsection{Modeling}

In light of the reflectance and far field characterizations results reported so far, the sensing probe reflectance of SENSOR 1 can be modeled as that of a Fabry Perot-type double interferometer while the behavior of SENSOR 2 cannot be trivially assumed like the one of a simple double interferometer. In case of SENSOR 1, the reflectance can be written according to the following equation:

$$
\begin{gathered}
R=\mid\left(r_{12}+r_{23} \cdot\left(1-A_{12}\right) \cdot e^{\mathbf{i} \cdot \beta_{\mathrm{HOF}}}+r_{34} \cdot\left(1-A_{12}\right) \cdot\left(1-A_{23}\right)\right. \\
\left.\cdot e^{\mathbf{i} \cdot\left(\boldsymbol{\beta}_{\mathrm{HOF}}+\boldsymbol{\beta}_{\mathrm{SWCNTS}}\right)}+r_{12} \cdot r_{23} \cdot r_{34} \cdot e^{\mathbf{i} \cdot \boldsymbol{\beta}_{\mathrm{SWCNTS}}}\right) \\
\div\left(1+r_{12} \cdot r_{23} \cdot e^{\mathbf{i} \cdot \boldsymbol{\beta}_{\mathrm{HOF}}}+r_{12} \cdot r_{34} \cdot\left(1-A_{23}\right)\right. \\
\cdot e^{\mathbf{i} \cdot\left(\boldsymbol{\beta}_{\mathrm{HOF}}+\boldsymbol{\beta}_{\mathrm{SWCNTS}}\right)}+\left.r_{23} \cdot r_{34} \cdot e^{\left.\mathbf{i} \cdot \boldsymbol{\beta}_{\mathrm{SWCNTS}}\right)}\right|^{2}
\end{gathered}
$$


with

$$
\begin{gathered}
r_{12}=\frac{n_{\mathrm{SOF}}-n_{\mathrm{HOF}}}{n_{\mathrm{SOF}}+n_{\mathrm{HOF}}}, \\
r_{23}=\frac{n_{\mathrm{HOF}}-n_{\mathrm{SWCNTs}}}{n_{\mathrm{HOF}}+n_{\mathrm{SWCNTs}}}, \\
r_{34}=\frac{n_{\mathrm{SWCNTs}}-n_{\mathrm{ext}}}{n_{\mathrm{SWCNTS}}+n_{\mathrm{ext}}}, \\
\beta_{\mathrm{HOF}}=\left(\frac{4 \cdot \pi}{\lambda}\right) \cdot n_{\mathrm{HOF}} \cdot d_{\mathrm{HOF}}, \\
\beta_{\mathrm{SWCNTS}}=\left(\frac{4 \cdot \pi}{\lambda}\right) \cdot n_{\mathrm{SWCNTs}} \cdot d_{\mathrm{SWCNTs}} .
\end{gathered}
$$

The symbols $r, n, d$, and $A$ represent, respectively, the reflection coefficient, the complex effective refractive index, the cavity length, and the absorbance (due to the coupling and splicing losses), while the subscript indices indicate the interface they refer to and the subscript labels indicate the referred cavity. In particular, the subscript 12 refers to the SOF-HOF interface, 23 to the HOF-SWCNTs interface, and 34 to the SWCNTs-external medium interface, while the subscript labels SOF, HOF, and SWCNTs have an obvious meaning. The reflectance $R$ is ruled by several factors involved in the propagation of the lightwave through the $\mathrm{HOF}$ and the HOF filled with SWCNTs. In particular, any change in the SWCNT refractive index affects the reflectance $R$ through the reflection coefficients $r_{23}$ and $r_{34}$, the absorbance $A_{23}$, and the propagation constant $\beta_{\text {SWCNTs }}$ according to (8) and (9).

It is noteworthy that the sensor sensitivity to the changes in the refractive index of the sensitive material used to functionalize the HOFs, which in turn are induced by its interaction with the chemicals under investigation, cannot be thought as derived by a simple Fabry-Perot effect in light of the PBG modifications. For instance, at the interface between the HOF and the HOF filled with SWCNTs, coupling losses due to the modal overlap between modes propagating in the sections with different guiding properties affect the reflectance $R$. This means that as the complex refractive index of the SWCNTs is modified by chemical sorption, consequent changes are expected in the coupling coefficients as well as in the reflectance $R$. On the other hand, also the propagation losses within the HOF region filled with SWCNTs depend upon the SWCNT refractive index and hence contribute to the reflectance variations.

In summary, the changes in the complex effective refractive index of SWCNTs within the filled region of HOF determine, in combination with the penetration depth of the carbon nanotubes, the sensing probe reflectance changes by affecting the propagation features of the sensing probe itself. This means that the key factor in the determination of the performance of this class of sensors relies in the possibility to exploit the aforementioned losses variability in order to get enhanced performance with respect to traditional SWCNTsbased SOF sensors.

\subsection{Experimental results}

In order to investigate the sensing capability of the fabricated HOF sensors, they have been placed in the test chamber schematically described in Section 5.1 and exposed to different concentrations of VOC vapors. At the same time, the sensors under test were interrogated with the same interrogation system exploited for the SOF sensors (described in detail in Section 3.2), allowing the continuous monitoring of the probe reflectance. It is noteworthy that since the light source used offers a bandwidth much wider than the separation of the faster interference fringes, the sensing performance exploited by each sensor basically depends on the slow features of the reflectance spectrum and thus mainly on the SWCNT cavity. During the measurements, the temperature within the chamber has been monitored by using a commercial thermocouple. The sensor sensitivity to temperature variations was previously characterized and used in order to properly compensate the sensor output. In particular, a comparison among the performances of SENSORS 1 and 2 is carried out by considering their $\Delta R / R_{0}$ due to toluene exposure (see Figure 36(a)). To this aim, both sensors have been exposed to three toluene pulses of 45 minutes with increasing concentrations in the ppm range.

On exposure, the reflectance of SENSOR 1 decreased linearly with the toluene concentration as confirmed by further exposure measurements resumed in Figure 36(b). Differently, SENSOR 2 reflectance increased upon exposure presenting a significantly lower and of opposite sign sensitivity. Moreover, SENSOR 2 turned out to be not able to reach a steady state within 45 minutes especially for high concentration exposures; this also limits the maximum reflectance change observed during the exposure tests.

The higher response times and the lower sensitivity of SENSOR 2 can be explained on the basis of the higher extension of the SWCNT region (higher diffusion times and higher losses in the filled region). In fact, the target VOC molecules able to interact with the SWCNTs lead to changes in the SWCNT agglomerate complex refractive index and the induced variations are detected as fast as short is the SWCNT region. In addition, as demonstrated by the far field characterization, the CNT penetration depth in SENSOR 2 makes the functionalized HOF not more able to guide the light and thus it reduces the capability of the light to interact with the sensitive material. Further investigation is required to assess the sensitivity dependence on the SWCNT distribution within the HOF structure and also to identify characterization features able to predict the correct functioning and the performance of the final device.

\section{CONCLUSION AND FUTURE PROSPECTS}

In conclusion, in this paper the combination of advanced nanostructured coatings based on single-walled carbon nanotubes with the optical fiber technology for the development of high-performance optochemical nanosensors has been reviewed. The sensitive material integration has been performed by the customization of the well-known molecular engineered Langmuir-Blodgett deposition technique that has 


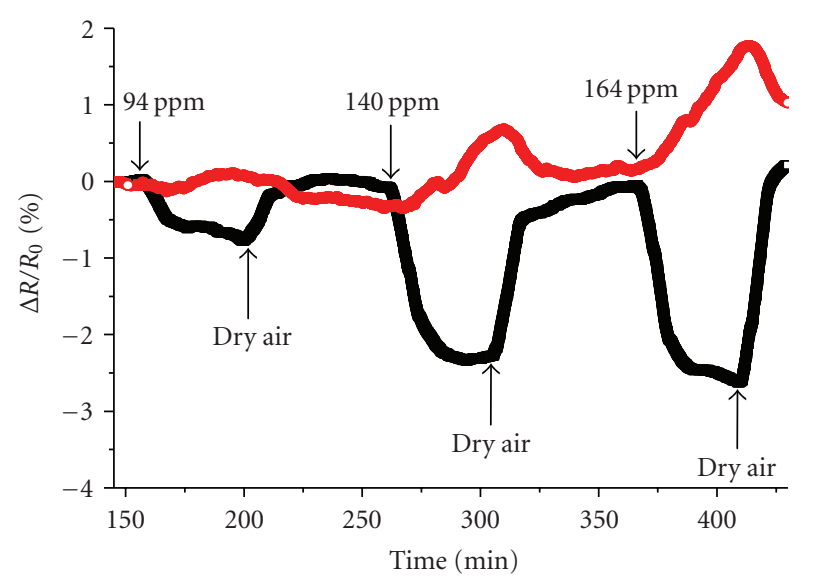

ーー Sensor 1

-०- Sensor 2

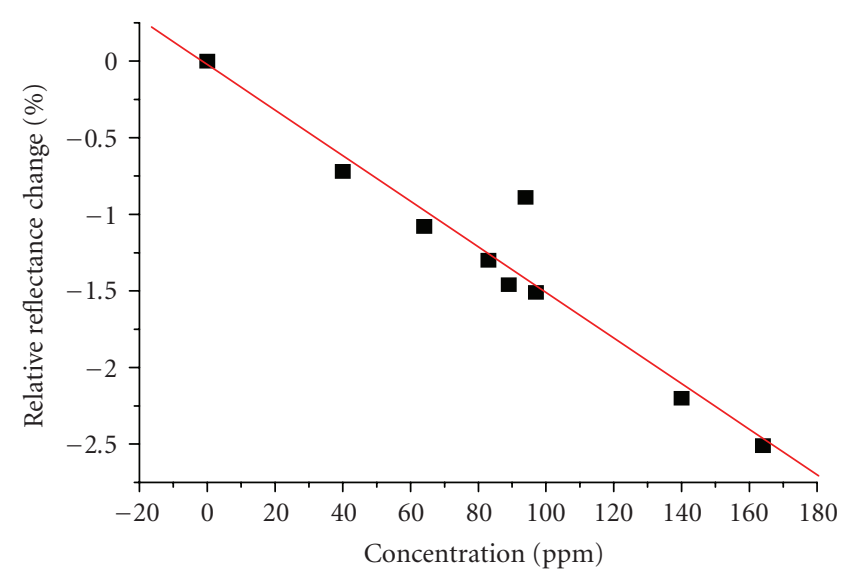

(b)

FIGURE 36: (a) Time responses of SENSORS 1 and 2 to three toluene vapor pulses, with concentrations in the range 84-164 ppm, at room temperature, (b) $\Delta R / R_{0}$ of SENSOR 1 corresponding to the steady states reached on exposure to different concentration pulses of toluene vapors.

been here used to transfer ultrathin layers based on SWCNTs upon the distal end of silica optical fibers. The adopted optical configuration is based on an extrinsic low-finesse FabryPerot interferometer, with its principle of operation relying, therefore, on the measurement of the changes in the amount of power reflected at the fiber-film interface occurring as a consequence of the changes in the optical (complex refractive index) and geometrical properties (thickness) of the sensitive elements. Such modifications are, in turn, caused by the interaction of the sensing layers with the target analyte molecules present in the environment.

Since the heart of a chemical sensor is the sensitive layer, from which the main performances of the sensing device are derived, a strong effort has been devoted to the investigation of the carbon nanotube overlays features. For this reason, extensive structural and morphological characterizations either of the as-bought SWCNT powders or of the deposited LB SWCNT films have been carried out by means of X-ray diffraction and Raman spectroscopy analyses, high-resolution transmission electron microscopy, and scanning electron microscopy observations. They confirmed the nanostructured dimensions of the exploited class of sensitive material as well as its successful transfer upon the optical fiber surface. The realized chemical sensors have been tested against VOCs and other pollutants in different conditions ranging from gaseous to aqueous environments, up to harsh environments at cryogenic temperatures suitable for space application. In most of the investigated cases, the fiber optic chemosensors coated by SWCNTs demonstrated their strong potentiality as well as the ability of detecting environmental pollutants around or well below the ppm threshold. The sensing performance of the proposed optochemical probes can be easily tailored by a proper choice of the number of SWCNT monolayers. This choice, however, has to be made by taking account of the tradeoff between sensor sensitivity and response time. Furthermore, the use of CdA/SWCNTs-based nanocomposites as novel sensitive nanocoatings has also been presented, revealing their strong potentiality to be successfully employed for chemical sensing both in liquid and gas phases. In addition, new advanced sensing configurations have been proposed, based on the use of hollow-core optical fibers coated and partially filled by carbon nanotubes.

\subsection{Future prospects}

As already discussed in Section 4.4, one of the major concerns with chemical sensors is how to improve their discrimination ability among different analytes. In fact, we are still far away from having synthetic and tunable materials able to mimic the tremendous molecular recognition capability of the biological receptors such as enzymes. However, there are two possible approaches in the attempt to increase the chemical sensor selectivity, and both should be followed in synergy. The first one is a direct approach which relies on the sensitive layer functionalization in order to have a higher affinity of the sensitive elements towards specific chemical species [82]. The second one is based on the use of a hybrid system composed of multiple transducers coated by different sensitive materials in the form of an array. Here, sensors with a poor selective response, when considered collectively, provide unique patterns typical for each analyte. The generated response patterns are interpreted by patternrecognition algorithm for the selective detection [83]. On this line of argument, the future work will be mainly devoted to the second solution. In particular, the use of chemical sensor arrays, based either on a single class of materials (SWCNTs) deposited on transducers with different operating principles, or on various types of materials (SWCNTs, nanoporous polymers, and metal oxides) [90-92] combined 
with a single type of transducers, or even on a multimaterial and multitransducers approach, will be investigated. At the same time, particular attention will be focused on the definition and application of suitable pattern recognition algorithms to interpret the results of the multielement arrays.

Further interest will also be devoted to the resolution of some unsolved matters, first of all to the improvement of the repeatability of the CNT overlay deposition process by means of their alignment upon the optical fiber surface. From this approach, an almost predictable distribution of the carbon tubes is expected, which should lead to an improved repeatability of the integration process. In addition, the optical anisotropy characteristics of such aligned carbon nanotubes overlays, first discovered at the optical communication wavelength in 2005 by Yoo et al. [93], could also be investigated and exploited for chemical sensing.

Future works will also deal with a deeper investigation of the dependence of the sensing performances of the SWCNTs composites-based transducers on the carbon nanotube filler content within the CdA host-matrix. In particular, since the content of SWCNTs can influence the sensitivity and dynamic characteristics of the composites-based sensors and since the sensitive area (i.e., the one corresponding to the core region of the fiber, which has a diameter of approx. $9 \mu \mathrm{m})$ is shorter than the one upon which the overlay is effectively deposited (the whole diameter of the fiber is of approx. $125 \mu \mathrm{m}$ ), the carbon nanotubes dispersion within the host material represent a challenging issue for which much attention should be paid.

Finally, appropriate integration procedures for the selective filling of the HOF holes and to control the SWCNT spatial distribution inside them are under investigation. This could lead to ad hoc modifications of the photonic bandgap offered by the functionalized hollow-core optical fibers, which could be very useful for the development of highperformance sensing configurations as well as new photonic devices.

\section{REFERENCES}

[1] B. B. Looney and R. W. Falta, Eds., Vadose Zone Science and Technology Solutions, Battelle Press, Columbus, Ohio, USA, 2000.

[2] L. G. Wilson, L. G. Everett, and S. J. Cullen, Eds., Handbook of Vadose Zone Characterization and Monitoring, CRC Press, Boca Raton, Fla, USA, 1995.

[3] J. W. Grate and M. H. Abraham, "Solubility interactions and the design of chemically selective sorbent coatings for chemical sensors and arrays," Sensors and Actuators B, vol. 3, no. 2, pp. 85-111, 1991.

[4] O. S. Wolfbeis, "Fiber-optic chemical sensors and biosensors," Analytical Chemistry, vol. 76, no. 12, pp. 3269-3284, 2004.

[5] L. J. Kepley, R. M. Crooks, and A. J. Ricco, "A selective SAWbased organophosphonate chemical sensor employing a selfassembled, composite monolayer: a new paradigm for sensor design," Analytical Chemistry, vol. 64, no. 24, pp. 3191-3193, 1992.

[6] M. Consales, S. Campopiano, A. Cutolo, et al., "Carbon nanotubes thin films fiber optic and acoustic VOCs sensors: performances analysis," Sensors and Actuators B, vol. 118, no. 1-2, pp. 232-242, 2006.
[7] M. Penza, G. Cassano, P. Aversa, et al., "Acoustic and optical VOCs sensors incorporating carbon nanotubes," IEEE Sensors Journal, vol. 6, no. 4, pp. 867-875, 2006.

[8] J. Hartmann, J. Auge, and P. Hauptmann, "Using the quartz crystal microbalance principle for gas detection with reversible and irreversible sensors," Sensors and Actuators B, vol. 19, no. 1-3, pp. 429-433, 1994.

[9] J. W. Grate, "Acoustic wave microsensor arrays for vapor sensing," Chemical Reviews, vol. 100, no. 7, pp. 2627-2648, 2000.

[10] M. Penza, G. Cassano, P. Aversa, et al., "Carbon nanotubescoated multi-transducing sensors for VOCs detection," Sensors and Actuators B, vol. 111-112, pp. 171-180, 2005.

[11] J. Zhang, J. Hu, Z. Q. Zhu, H. Gong, and S. J. O'Shea, “Quartz crystal microbalance coated with sol-gel-derived indium-tin oxide thin films as gas sensor for NO detection," Colloids and Surfaces A, vol. 236, no. 1-3, pp. 23-30, 2004.

[12] D. James, S. M. Scott, Z. Ali, and W. T. O'Hare, "Chemical sensors for electronic nose systems," Microchimica Acta, vol. 149, no. 1-2, pp. 1-17, 2005.

[13] F. Baldini, A. N. Chester, J. Homola, and S. Martellucci, Optical Chemical Sensors, vol. 224 of NATO Science Series II: Mathematics, Physics and Chemistry, Springer, Berlin, Germany, 2006.

[14] D. W. Lübbers and N. Opitz, "Eine neue $\mathrm{pCO}_{2}$-bzw. $\mathrm{pO}_{2}$ Messsonde zur Messung des $\mathrm{pCO}_{2}$ oder $\mathrm{pO}_{2}$ von Gasen und Flüssigkeiten," Zeitschrift für Naturforschung C, vol. 30, no. 4, pp. 532-533, 1975.

[15] I. Zudans, W. R. Heineman, and C. J. Seliskar, "In situ measurements of chemical sensor film dynamics by spectroscopic ellipsometry. Three case studies," Thin Solid Films, vol. 455456, pp. 710-715, 2004.

[16] I. M. Steinberg, A. Lobnik, and O. S. Wolfbeis, "Characterisation of an optical sensor membrane based on the metal ion indicator Pyrocatechol Violet," Sensors and Actuators B, vol. 90, no. 1-3, pp. 230-235, 2003.

[17] G. Orellana, "Luminescent optical sensors," Analytical and Bioanalytical Chemistry, vol. 379, no. 3, pp. 344-346, 2004.

[18] J. Homola, S. S. Yee, and G. Gauglitz, "Surface plasmon resonance sensors: review," Sensors and Actuators B, vol. 54, no. 1, pp. 3-15, 1999.

[19] A. G. Mignani, L. Ciaccheri, A. Cimato, C. Attilio, and P. R. Smith, "Spectral nephelometry for the geographic classification of Italian extra virgin olive oils," Sensors and Actuators B, vol. 111-112, pp. 363-369, 2005.

[20] A. G. Mignani, A. A. Mencaglia, and L. Ciaccheri, "Fiber optic systems for colorimetry and scattered colorimetry," in Optical Fibers: Applications, vol. 5952 of Proceedings of the SPIE, pp. 89-99, Warsaw, Poland, September 2005.

[21] F. J. Arregui, I. R. Matías, and R. O. Claus, "Optical fiber gas sensors based on hydrophobic alumina thin films formed by the electrostatic self-assembly monolayer process," IEEE Sensors Journal, vol. 3, no. 1, pp. 56-61, 2003.

[22] O. S. Wolfbeis, Fiber Optic Chemical Sensors and Biosensors. Vol. II, CRC Press, Boca Raton, Fla, USA, 1991.

[23] A. Brecht and G. Gauglitz, "Optical probes and transducers," Biosensors and Bioelectronics, vol. 10, no. 9-10, pp. 923-936, 1995.

[24] G. Gauglitz, Opto-Chemical and Opto-Immuno Sensors, vol. 1 of Sensor Update, VCH Verlagsgesellschaft, Weinheim, Germany, 1996.

[25] G. Boisde and A. Harmer, Chemical and Biochemical Sensing with Optical Fibers and Waveguides, Artech House, Boston, Mass, USA, 1996. 
[26] X.-J. Huang and Y.-K. Choi, "Chemical sensors based on nanostructured materials," Sensors and Actuators B, vol. 122, no. 2, pp. 659-671, 2007.

[27] S. Iijima, "Helical microtubules of graphitic carbon," Nature, vol. 354, no. 6348, pp. 56-58, 1991.

[28] M. S. Dresselhaus, G. Dresselhaus, and Ph. Avouris, Carbon Nanotubes: Synthesis, Structure, Properties, and Applications, vol. 80 of Topics in Applied Physics, Springer, Berlin, Germany, 2001.

[29] H. Dai, "Carbon nanotubes: synthesis, integration, and properties," Accounts of Chemical Research, vol. 35, no. 12, pp. 1035-1044, 2002.

[30] M. Terrones, "Science and technology of the twenty-first century: synthesis, properties, and applications of carbon nanotubes," Annual Review of Materials Research, vol. 33, pp. 419-501, 2003.

[31] M. Dresselhaus, G. Dresselhaus, and P. C. Eklund, Science of Fullerenes and Carbon Nanotubes, Academic Press, San Diego, Calif, USA, 1996.

[32] J. Riu, A. Maroto, and F. X. Rius, "Nanosensors in environmental analysis," Talanta, vol. 69, no. 2, pp. 288-301, 2006.

[33] A. Modi, N. Koratkar, E. Lass, B. Wei, and P. M. Ajayan, "Miniaturized gas ionization sensors using carbon nanotubes," Nature, vol. 424, no. 6945, pp. 171-174, 2003.

[34] J. Kong, N. R. Franklin, C. Zhou, et al., "Nanotube molecular wires as chemical sensors," Science, vol. 287, no. 5453, pp. 622625, 2000.

[35] P. G. Collins, K. Bradley, M. Ishigami, and A. Zettl, "Extreme oxygen sensitivity of electronic properties of carbon nanotubes," Science, vol. 287, no. 5459, pp. 1801-1804, 2000.

[36] G. U. Sumanasekera, C. K. W. Adu, S. Fang, and P. C. Eklund, "Effects of gas adsorption and collisions on electrical transport in single-walled carbon nanotubes," Physical Review Letters, vol. 85, no. 5, pp. 1096-1099, 2000.

[37] A. Zahab, L. Spina, P. Poncharal, and C. Marlière, "Watervapor effect on the electrical conductivity of a single-walled carbon nanotube mat," Physical Review B, vol. 62, no. 15, pp. 10000-10003, 2000.

[38] A. Fujiwara, K. Ishii, H. Suematsu, et al., "Gas adsorption in the inside and outside of single-walled carbon nanotubes," Chemical Physics Letters, vol. 336, no. 3-4, pp. 205-211, 2001.

[39] C. K. W. Adu, G. U. Sumanasekera, B. K. Pradhan, H. E. Romero, and P. C. Eklund, "Carbon nanotubes: a thermoelectric nano-nose," Chemical Physics Letters, vol. 337, no. 1-3, pp. 31-35, 2001.

[40] G. U. Sumanasekera, B. K. Pradhan, C. K. W. Adu, H. E. Romero, H. C. Foley, and P. C. Eklund, "Thermoelectric chemical sensor based on single wall carbon nanotubes," Molecular Crystals and Liquid Crystals, vol. 387, no. 1, pp. 3137, 2002.

[41] S. Chopra, K. McGuire, N. Gothard, A. M. Rao, and A. Pham, "Selective gas detection using a carbon nanotube sensor," Applied Physics Letters, vol. 83, no. 11, pp. 2280-2282, 2003.

[42] B.-Y. Wei, C.-S. Lin, and H.-M. Lin, "Examining the gassensing behaviors of carbon nanotubes using a piezoelectric quartz crystal microbalance," Sensors and Materials, vol. 15, no. 4, pp. 177-190, 2003.

[43] M. Penza, F. Antolini, and M. V. Antisari, "Carbon nanotubes as SAW chemical sensors materials," Sensors and Actuators B, vol. 100, no. 1-2, pp. 47-59, 2004.

[44] R. J. Chen, S. Bangsaruntip, K. A. Drouvalakis, et al., "Noncovalent functionalization of carbon nanotubes for highly specific electronic biosensors," Proceedings of the National
Academy of Sciences of the United States of America, vol. 100, no. 9, pp. 4984-4989, 2003.

[45] B. R. Azamian, J. J. Davis, K. S. Coleman, C. B. Bagshaw, and M. L. H. Green, "Bioelectrochemical single-walled carbon nanotubes," Journal of the American Chemical Society, vol. 124, no. 43, pp. 12664-12665, 2002.

[46] Q. Zhao, M. D. Frogley, and H. D. Wagner, "Directionsensitive stress measurements with carbon nanotube sensors," Polymers for Advanced Technologies, vol. 13, no. 10-12, pp. 759-764, 2002.

[47] S. Sotiropoulou, V. Gavalas, V. Vamvakaki, and N. A. Chaniotakis, "Novel carbon materials in biosensor systems," Biosensors and Bioelectronics, vol. 18, no. 2-3, pp. 211-215, 2003.

[48] P. W. Barone, S. Baik, D. A. Heller, and M. S. Strano, "Near-infrared optical sensors based on single-walled carbon nanotubes," Nature Materials, vol. 4, no. 1, pp. 86-92, 2005.

[49] A. Cusano, M. Consales, A. Cutolo, et al., "Optical probes based on optical fibers and single-walled carbon nanotubes for hydrogen detection at cryogenic temperatures," Applied Physics Letters, vol. 89, no. 20, Article ID 201106, 3 pages, 2006.

[50] M. Penza, G. Cassano, P. Aversa, et al., "Carbon nanotube acoustic and optical sensors for volatile organic compound detection," Nanotechnology, vol. 16, no. 11, pp. 2536-2547, 2005.

[51] M. Penza, G. Cassano, P. Aversa, et al., "Alcohol detection using carbon nanotubes acoustic and optical sensors," Applied Physics Letters, vol. 85, no. 12, pp. 2379-2381, 2004.

[52] M. Consales, A. Crescitelli, S. Campopiano, et al., "Chemical detection in water by single-walled carbon nanotubes-based optical fiber sensors," IEEE Sensors Journal, vol. 7, no. 7, pp. 1004-1005, 2007.

[53] A. Cusano, M. Pisco, M. Consales, et al., "Novel optochemical sensors based on hollow fibers and single walled carbon nanotubes," IEEE Photonics Technology Letters, vol. 18, no. 22, pp. 2431-2433, 2006.

[54] C. Fabry and A. Perot, "Théorie et applications dune novelle méthode de spectroscopie interférentielle," Annales de Chimie et de Physique, vol. 16, pp. 115-144, 1899.

[55] J. Dakin and B. Culshaw, Optical Fiber Sensors: Principle and Components, Artech House, Boston, Mass, USA, 1988.

[56] D. A. Jackson, "Recent progress in monomode fibre-optic sensors," Measurement Science and Technology, vol. 5, no. 6, pp. 621-638, 1994.

[57] M. A. Chan, S. D. Collins, and R. L. Smith, "A micromachined pressure sensor with fiber-optic interferometric readout," Sensors and Actuators A, vol. 43, no. 1-3, pp. 196-201, 1994.

[58] H. A. Macleod, Thin Film Optical Filters, Institute of Physics, Philadelphia, Pa, USA, 3rd edition, 2001.

[59] A. D. Kersey and A. Dandridge, "Applications of fiber-optic sensors," IEEE Transactions on Components, Hybrids, and Manufacturing Technology, vol. 13, no. 1, pp. 137-143, 1990.

[60] A. Bachtold, P. Hadley, T. Nakanishi, and C. Dekker, "Logic circuits with carbon nanotube transistors," Science, vol. 294, no. 5545, pp. 1317-1320, 2001.

[61] D. Y. Takamoto, E. Aydil, J. A. Zasadzinski, et al., "Stable ordering in Langmuir-Blodgett films," Science, vol. 293, no. 5533, pp. 1292-1295, 2001.

[62] T. Di Luccio, F. Antolini, P. Aversa, G. Scalia, and L. Tapfer, "Structural and morphological investigation of LangmuirBlodgett SWCNT/behenic acid multilayers," Carbon, vol. 42, no. 5-6, pp. 1119-1122, 2004. 
[63] G. Roberts, Langmuir-Blodgett Films, Plenum Press, New York, NY, USA, 1990.

[64] S. W. James and R. P. Tatam, "Fibre optic sensors with nanostructured coatings," Journal of Optics A, vol. 8, no. 7, pp. S430-S444, 2006.

[65] C. Journet, W. K. Maser, P. Bernier, et al., "Large-scale production of single-walled carbon nanotubes by the electricarc technique," Nature, vol. 388, no. 6644, pp. 756-758, 1997.

[66] Y. Saito, Y. Tani, N. Miyagawa, K. Mitsushima, A. Kasuya, and Y. Nishina, "High yield of single-wall carbon nanotubes by arc discharge using Rh-Pt mixed catalysts," Chemical Physics Letters, vol. 294, no. 6, pp. 593-598, 1998.

[67] R. Saito, G. Dresselhaus, and M. S. Dresselhaus, Physical Properties of Carbon Nanotubes, Imperial College Press, London, UK, 1998.

[68] M. Consales, S. Campopiano, A. Cutolo, et al., "Sensing properties of buffered and not buffered carbon nanotubes by fibre optic and acoustic sensors," Measurement Science and Technology, vol. 17, no. 5, pp. 1220-1228, 2006.

[69] A. Cusano, G. V. Persiano, M. Russo, and M. Giordano, "Novel optoelectronic sensing system for thin polymer films glass transition investigation," IEEE Sensors Journal, vol. 4, no. 6, pp. 837-844, 2004.

[70] A. D'Amico and C. Di Natale, "A contribution on some basic definitions of sensors properties," IEEE Sensors Journal, vol. 1, no. 3, pp. 183-190, 2001.

[71] A. Abdelghani and N. Jaffrezic-Renault, "SPR fibre sensor sensitised by fluorosiloxane polymers," Sensors and Actuators $B$, vol. 74, no. 1-3, pp. 117-123, 2001.

[72] F. Abdelmalek, J. M. Chovelon, M. Lacroix, N. JaffrezicRenault, and V. Matejec, "Optical fibre sensors sensitized by phenyl-modified porous silica prepared by sol-gel," Sensors and Actuators B, vol. 56, no. 3, pp. 234-242, 1999.

[73] I. Lundström, S. Shivaraman, C. Svensson, and L. Lundkvist, "A hydrogen-sensitive MOS field-effect transistor," Applied Physics Letters, vol. 26, no. 2, pp. 55-57, 1975.

[74] I. Lundström, "Hydrogen sensitive mos-structures-part 1: principles and applications," Sensors and Actuators, vol. 1, pp. 403-426, 1981.

[75] M. A. Butler, "Micromirror optical-fiber hydrogen sensor," Sensors and Actuators B, vol. 22, no. 2, pp. 155-163, 1994.

[76] B. Sutapun, M. Tabib-Azar, and A. Kazemi, "Pd-coated elastooptic fiber optic Bragg grating sensors for multiplexed hydrogen sensing," Sensors and Actuators B, vol. 60, no. 1, pp. 27-34, 1999.

[77] M. A. Butler, "Fiber optic sensor for hydrogen concentrations near the explosive limit," Journal of the Electrochemical Society, vol. 138, no. 9, pp. L46-L47, 1991.

[78] J. Villatoro, A. Díez, J. L. Cruz, and M. V. Andrés, "Highly sensitive optical hydrogen sensor using circular Pd-coated singlemode tapered fibre," Electronics Letters, vol. 37, no. 16, pp. 1011-1012, 2001.

[79] A. Güemes, J. M. Pintado, M. Frövel, E. Olmo, and A. Obst, "Comparison of three types of fibre optic hydrogen sensors within the frame of CryoFOS project," in 17th International Conference on Optical Fibre Sensors, M. Voet, R. Willsch, W. Ecke, J. Jones, and B. Culshaw, Eds., vol. 5855 of Proceedings of SPIE, pp. 1000-1003, Bruges, Belgium, May 2005.

[80] H. Takagi, H. Hatori, Y. Soneda, N. Yoshizawa, and Y. Yamada, "Adsorptive hydrogen storage in carbon and porous materials," Materials Science and Engineering B, vol. 108, no. 1-2, pp. 143-147, 2004.
[81] A. C. Dillon, K. M. Jones, T. A. Bekkedahl, C. H. Kiang, D. S. Bethune, and M. J. Heben, "Storage of hydrogen in singlewalled carbon nanotubes," Nature, vol. 386, no. 6623, pp. 377379, 1997.

[82] K. Balasubramanian and M. Burghard, "Chemically functionalized carbon nanotubes,” Small, vol. 1, no. 2, pp. 180-192, 2005.

[83] S. Zaromb and J. R. Stetter, "Theoretical basis for identification and measurement of air contaminants using an array of sensors having partially overlapping sensitivities," Sensors and Actuators, vol. 6, no. 4, pp. 225-243, 1984.

[84] M. Penza, M. A. Tagliente, P. Aversa, G. Cassano, and L. Capodieci, "Single-walled carbon nanotubes nanocomposite microacoustic organic vapor sensors," Materials Science and Engineering C, vol. 26, no. 5-7, pp. 1165-1170, 2006.

[85] P. Mach, M. Dolinski, K. W. Baldwin, et al., "Tunable microfluidic optical fiber," Applied Physics Letters, vol. 80, no. 23, pp. 4294-4296, 2002.

[86] Y. Huang, Y. Xu, and A. Yariv, "Fabrication of functional microstructured optical fibers through a selective-filling technique," Applied Physics Letters, vol. 85, no. 22, pp. 5182-5184, 2004.

[87] S. A. Cerqueira Jr., F. Luan, C. M. B. Cordeiro, A. K. George, and J. C. Knight, "Hybrid photonic crystal fiber," Optics Express, vol. 14, no. 2, pp. 926-931, 2006.

[88] M. Pisco, M. Consales, A. Cutolo, A. Cusano, M. Penza, and P. Aversa, "Hollow fibers integrated with single walled carbon nanotubes: bandgap modification and chemical sensing capability," Sensors and Actuators B, vol. 129, no. 1, pp. 163-170, 2008.

[89] J. C. Manifacier, J. Gasiot, and J. P. Fillard, "A simple method for the determination of the optical constants $n, k$ and the thickness of a weakly absorbing thin film," Journal of Physics E, vol. 9, no. 11, pp. 1002-1004, 1976.

[90] M. Giordano, M. Russo, A. Cusano, and G. Mensitieri, "An high sensitivity optical sensor for chloroform vapours detection based on nanometric film of $\delta$-form syndiotactic polystyrene," Sensors and Actuators B, vol. 107, no. 1, pp. 140147, 2005.

[91] A. Cusano, M. Consales, M. Pisco, et al., "Optochemical sensor for water monitoring based on $\mathrm{SnO}_{2}$ particle layer deposited onto optical fibers by the electrospray pyrolysis method," Applied Physics Letters, vol. 89, no. 11, Article ID 111103, 3 pages, 2006.

[92] M. Pisco, M. Consales, S. Campopiano, et al., "A novel optochemical sensor based on $\mathrm{SnO}_{2}$ sensitive thin film for ppm ammonia detection in liquid environment," Journal of Lightwave Technology, vol. 24, no. 12, pp. 5000-5007, 2006.

[93] S. Yoo, Y. Jung, D. S. Lee, et al., "Optical anisotropy in singlewalled carbon nanotubes," Optics Letters, vol. 30, no. 23, pp. 3201-3203, 2005. 

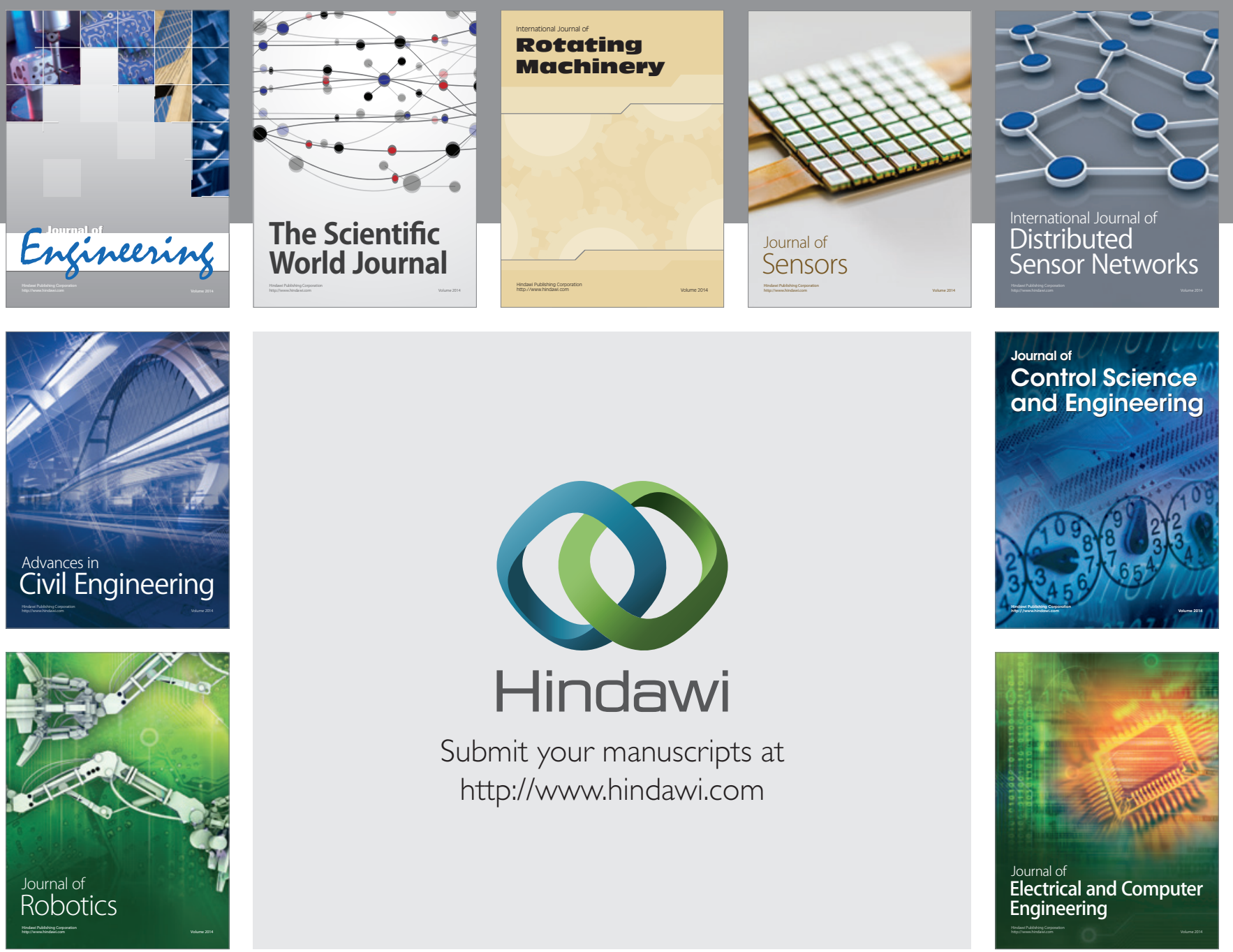

Submit your manuscripts at

http://www.hindawi.com
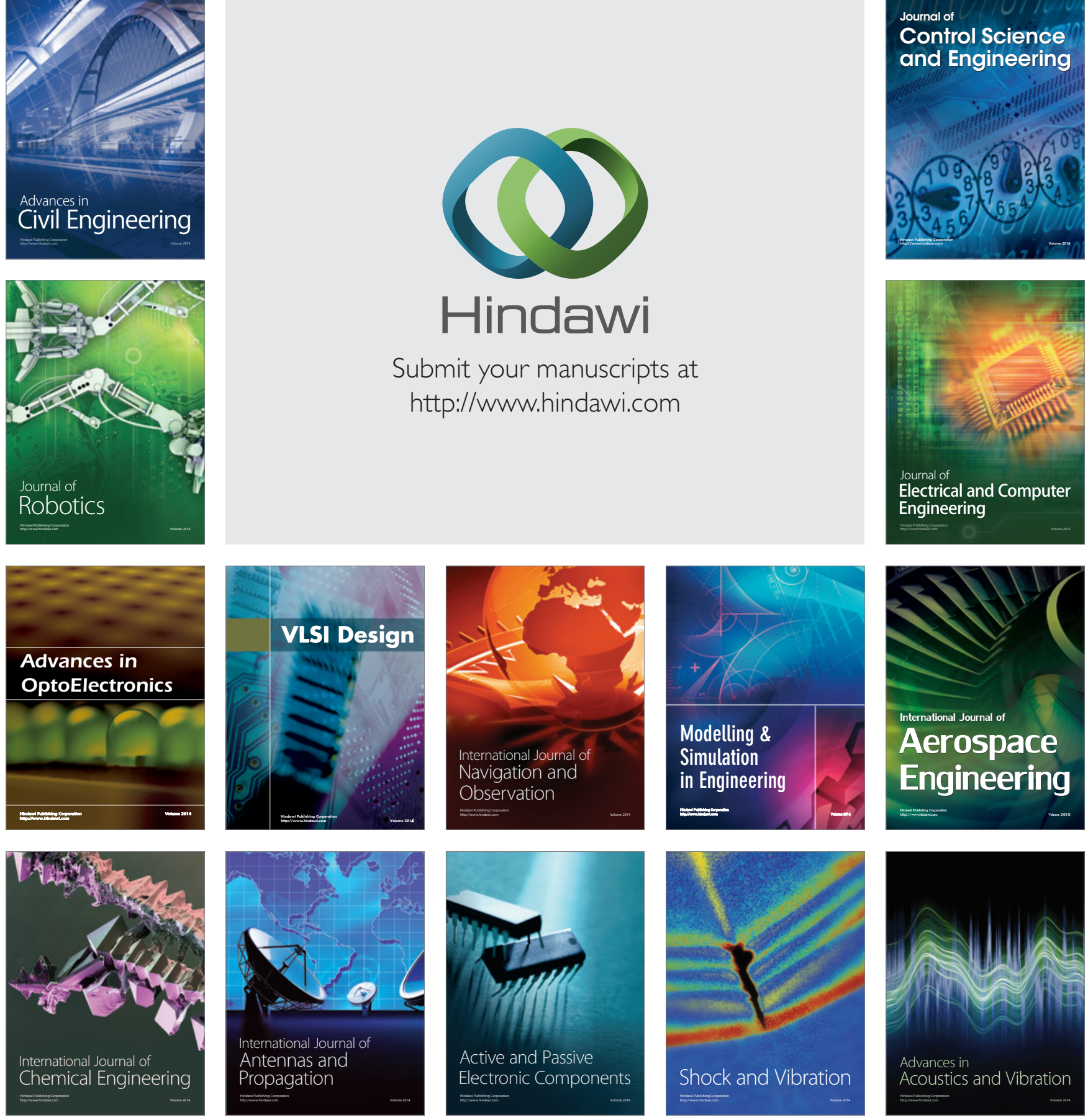